TRANSACTIONS OF THE

AMERICAN MATHEMATICAL SOCIETY

Volume 354, Number 8 , Pages 3275-3298

S 0002-9947(02)03001-5

Article electronically published on April 3, 2002

\title{
ON A CLASS OF JOINTLY HYPONORMAL TOEPLITZ OPERATORS
}

\author{
CAIXING GU
}

\begin{abstract}
We characterize when a pair of Toeplitz operators $\mathbf{T}=\left(T_{\phi}, T_{\psi}\right)$ is jointly hyponormal under various assumptions - for example, $\phi$ is analytic or $\phi$ is a trigonometric polynomial or $\phi-\psi$ is analytic. A typical characterization states that $\mathbf{T}=\left(T_{\phi}, T_{\psi}\right)$ is jointly hyponormal if and only if an algebraic relation of $\phi$ and $\psi$ holds and the single Toeplitz operator $T_{\omega}$ is hyponormal, where $\omega$ is a combination of $\phi$ and $\psi$. More general results for an $n$-tuple of Toeplitz operators are also obtained.
\end{abstract}

\section{INTRODUCTION}

Let $\mathcal{H}$ be a complex separable Hilbert space and let $B(\mathcal{H})$ be the algebra of bounded linear operators on $\mathcal{H}$. For $T, S \in B(\mathcal{H})$, the commutator of $T$ and $S$ is

$$
[T, S]:=T S-S T \text {. }
$$

An operator $T$ is hyponormal if the self-commutator $\left[T^{*}, T\right]=T^{*} T-T T^{*}$ is positive. Let $\mathbf{A}=\left(A_{1}, A_{2}, \cdots, A_{n}\right)$ be an $n$-tuple of operators on $\mathcal{H}$. The tuple $\mathbf{A}$ is jointly hyponormal (or simply, hyponormal) if the self-commutator $\left[\mathbf{A}^{*}, \mathbf{A}\right]$ of $\mathbf{A}$, defined by $\left[\mathbf{A}^{*}, \mathbf{A}\right]=\left(\left[A_{j}{ }^{*}, A_{i}\right]\right)$, is a positive operator on $\mathcal{H} \oplus \cdots \oplus \mathcal{H}$. This notion of joint hyponormality was first introduced by Athavale [3] in 1988 . We say $\mathbf{A}$ is weakly hyponormal if $\alpha_{1} A_{1}+\alpha_{2} A_{2}+\cdots \alpha_{n} A_{n}$ is hyponormal for all constants $\alpha_{1}, \cdots, \alpha_{n}$. It was observed in 3 that if $\mathbf{A}$ is jointly hyponormal then $\mathbf{A}$ is weakly hyponormal. These two notions are in general different even for commuting tuples, see [7, [15]. Joint hyponormality has been studied by several authors [3], 7], [11, [12], [14], [21].

In this paper we will study the joint hyponormality of Toeplitz operators. Let $L^{2}$ be the space of Lebesgue square integrable functions on the unit circle, and $L^{\infty}$ the space of essentially bounded functions on the unit circle. The Hardy space $H^{2}$ is the closed linear span of analytic polynomials in $L^{2}$. Let $P$ be the projection of $L^{2}$ onto $H^{2}$. For $\phi \in L^{\infty}$, the Toeplitz operator $T_{\phi}$ with symbol $\phi$ on the Hardy space $H^{2}$ is defined by the rule

$$
T_{\phi} h=P(\phi h) .
$$

Received by the editors December 28, 1999 and, in revised form, February 9, 2001 and December 3, 2001.

2000 Mathematics Subject Classification. Primary 47B35, 47B20.

Key words and phrases. Toeplitz operator, Hankel operator, joint hyponormality.

Partially supported by the National Science Foundation Grant DMS-9706838.

(C)2002 American Mathematical Society 
The unilateral shift $U$ is the Toeplitz operator $T_{z}$. The normality, subnormality and hyponormality of a single Toeplitz operator have been discussed in [1], [2], [6], [8], [9], [10] [16], 19], 22]. In particular, Cowen [8] gave an elegant characterization of hyponormal Toeplitz operators.

The study of jointly hyponormal Toeplitz operators started with the interesting observation, made by Farenick and MaEachin [17, that the joint hyponormality of $(U, T)$ implies that $T$ is necessarily a Toeplitz operator; see Proposition 2.3 below. They went on to characterize when $(U, T)$ is hyponormal. Let $\psi \in L^{\infty}$. Recently Curto and Lee 13 studied the joint hyponormality of the Toeplitz pair $\mathbf{T}=\left(T_{\phi}, T_{\psi}\right)$ when both symbols $\psi$ and $\phi$ are trigonometric polynomials. A complete characterization of hyponormal Toeplitz pairs in this case was given. Moreover, they showed that the weak hyponormality and the hyponormality of $\mathbf{T}$ are equivalent properties when both symbols are trigonometric polynomials.

In this paper we give an example of $\mathbf{T}=\left(T_{\phi}, T_{\psi}\right)$ where $\phi$ and $\psi$ are rational functions such that $\mathbf{T}$ is weakly hyponormal but not hyponormal. This will be done in Section 3 after some preliminary results in Section 2. This is achieved by noting that the weak hyponormality of $\mathbf{T}=\left(T_{\phi}, T_{\psi}\right)$ for a certain class of rational symbols is equivalent to the classical Hermite-Fejér interpolation problem via Cowen's characterization. For a single Toeplitz operator, this connection was observed by the author [19.

In Section 4, we characterize when $\mathbf{T}=\left(T_{\phi_{1}}, \cdots, T_{\phi_{n}}, T_{\psi}\right)$ is hyponormal for the case $\phi_{i} \in H^{\infty}$ for $i=1, \cdots, n$ and $\psi \in L^{\infty}$. This extends the result of [17], where it was assumed that $n=1$ and $\phi_{1}=z$, and the result of [13], where it was assumed that $n=1$ and $\phi_{1}$ is an analytic polynomial. This also allows us to construct a tuple of three Toeplitz operators $\mathbf{T}=\left(T_{\phi_{1}}, T_{\phi_{2}}, T_{\phi_{3}}\right)$ for which each subpair is hyponormal but $\mathbf{T}$ is not hyponormal, which gives a negative answer to a conjecture of Curto and Lee [13]. Indeed, it was shown in [13] that $\mathbf{T}=\left(T_{\phi_{1}}, \cdots, T_{\phi_{n}}\right)$ is hyponormal if and only if each subpair is hyponormal when all the $\phi_{i}$ are assumed to be trigonometric polynomials. The examples above indicate that when we deal with symbols more general than trigonometric polynomials, new phenomena occur. In particular, the technique of using weak hyponormality to study hyponormality as in 13 is not available, and the extension of the results for a pair of Toeplitz operators to a tuple of Toeplitz operators requires much more work.

In Section 5 we study the hyponormality of $\mathbf{T}=\left(T_{\phi_{1}}, \cdots, T_{\phi_{n}}\right)$ when all the $\phi_{i}$ have the same anti-analytic parts. We make some observations in Section 6 and give various sufficient and necessary conditions for the hyponormality of $\mathbf{T}=$ $\left(T_{\phi_{1}}, \cdots, T_{\phi_{n}}\right)$. In particular, we give a complete characterization of the hyponormality of $\mathbf{T}=\left(T_{\phi_{1}}, \cdots, T_{\phi_{n}}\right)$ when only one of them is assumed to have a trigonometric polynomial symbol. A typical characterization states that $\mathbf{T}=\left(T_{\phi}, T_{\psi}\right)$ is hyponormal if and only if a certain algebraic relation of $\phi$ and $\psi$ holds and a single Toeplitz operator whose symbol is a variation of $\psi$ or $\phi$ is hyponormal.

The approach we take is quite different from the ones in [17] and [13, where the proofs often rely on intricate and explicit computations using the coefficients of trigonometric polynomials involved. For more general symbols, explicit computation is very difficult, if not impossible. Indeed, here most computations are done at the operator and function levels in a more abstract way. Besides yielding more general results, our proofs seem to be shorter and more insightful. This is made possible by systematic use of Hankel operators and by avoiding direct computation of the inverses of certain operators. 


\section{SOME PRELIMINARY RESUlts}

We first establish some results for positive 2 by 2 block matrices. Let $\mathcal{H}$ and $\mathcal{G}$ be two separable complex Hilbert spaces. Let

$$
M(A, B, C)=\left(\begin{array}{cc}
A & B \\
B^{*} & C
\end{array}\right) \quad \text { on } \quad \mathcal{H} \oplus \mathcal{G}
$$

where $A, B$ and $C$ are operators acting between appropriate spaces. Let $\operatorname{Ker}(A)$, $\operatorname{Range}(A)$ and $\operatorname{Rank}(A)$ be the kernel, range and rank of $A$. For an operator $A$, the Moore-Penrose inverse $A^{\#}$ of $A$ is an operator satisfying

$$
A A^{\#} A=A, \quad A^{\#} A A^{\#}=A^{\#}, \quad\left(A A^{\#}\right)^{*}=A A^{\#}, \quad\left(A^{\#} A\right)^{*}=A^{\#} A .
$$

An operator on a Hilbert space has a Moore-Penrose inverse if and only if it has closed range, and the Moore-Penrose inverse is unique whenever it exists. In particular, if $A$ is positive and has closed range, and we write

$$
A=\left(\begin{array}{cc}
A_{0} & 0 \\
0 & 0
\end{array}\right): \operatorname{Range}(A) \oplus \operatorname{Ker}(A) \rightarrow \operatorname{Range}(A) \oplus \operatorname{Ker}(A),
$$

then the Moore-Penrose inverse $A^{\#}$ of $A$ is defined by

$$
A^{\#}=\left(\begin{array}{cc}
A_{0}^{-1} & 0 \\
0 & 0
\end{array}\right): \operatorname{Range}(A) \oplus \operatorname{Ker}(A) \rightarrow \operatorname{Range}(A) \oplus \operatorname{Ker}(A) .
$$

Lemma 2.1. a) $M(A, B, C)$ is positive if and only if both $A$ and $C$ are positive and

$$
|(h, B g)|^{2} \leq(A h, h)(C g, g) \quad \text { for all } h \in \mathcal{H}, g \in \mathcal{G} .
$$

b) If $M(A, B, C)$ is positive, then

$$
\operatorname{Ker}\left(B^{*}\right) \supset \operatorname{Ker}(A) \quad \text { and } \operatorname{Ker}(B) \supset \operatorname{Ker}(C) .
$$

In particular, if $A=0$, then $B=0$.

c) If Range $(A)$ is closed, let $A^{\#}$ be the Moore-Penrose inverse of $A$. Then $M(A, B, C)$ is positive if and only if both $A$ and $C$ are positive,

$$
\operatorname{Ker}\left(B^{*}\right) \supset \operatorname{Ker}(A) \quad \text { and } \quad C \geq B^{*} A^{\#} B .
$$

d) If $M(A, B, C)$ is positive and both $A$ and $C$ are of finite rank, then $M(A, B, C)$ is of finite rank and

$$
\operatorname{Rank}[M(A, B, C)]=\operatorname{Rank}(A)+\operatorname{Rank}\left(C-B^{*} A^{\#} B\right) .
$$

Proof. The proof is essentially from [14]. $M(A, B, C)$ is positive if and only if

$$
\langle M(A, B, C)(t h \oplus g),(t h \oplus g)\rangle \geq 0 \quad \text { for all } h \in \mathcal{H}, g \in \mathcal{G}
$$

and any real number $t$. Equivalently,

$$
t^{2}(A h, h)+2 t R e(h, B g)+(C g, g) \geq 0 .
$$

Analyzing the above quadratic function of $t$ yields the result in part a).

Part b) clearly follows from part a). Part c) is well known in the case when $A$ is invertible. Part c) in this general form also appeared in [13]. Here we give a slightly more direct proof, which is an adaptation of the proof for the invertible case and also yields the rank formula in part d). 
We next prove the sufficiency in part c). Let $\mathcal{H}_{0}$ be the range of $A$. It is easy to see that $A A^{\#}=A^{\#} A=P_{\mathcal{H}_{0}}$ The relation $\operatorname{Ker}\left(B^{*}\right) \supset \operatorname{Ker}(A)=\operatorname{Ker}\left(P_{\mathcal{H}_{0}}\right)$ implies that

$$
-B^{*} A^{\#} A+B^{*}=B^{*}\left(I-P_{\mathcal{H}_{0}}\right)=0 .
$$

Therefore,

$$
\begin{aligned}
& \left(\begin{array}{cc}
I & 0 \\
-B^{*} A^{\#} & I
\end{array}\right)\left(\begin{array}{cc}
A & B \\
B^{*} & C
\end{array}\right)\left(\begin{array}{cc}
I & -A^{\#} B \\
0 & I
\end{array}\right) \\
& =\left(\begin{array}{cc}
A & B-A A^{\#} B \\
-B^{*} A^{\#} A+B^{*} & C-B^{*} A^{\#} B
\end{array}\right)=\left(\begin{array}{cc}
A & 0 \\
0 & C-B^{*} A^{\#} B
\end{array}\right) .
\end{aligned}
$$

The sufficiency in part c) follows from the above matrix identity. The necessity is also clear by part b) and the above matrix identity.

The following lemma provides a contrast between the weak hyponormality and hyponormality of $\mathbf{A}=\left(A_{1}, A_{2}\right)$. The first part is Lemma 1.4 in 14 and the second part is Proposition 2.5 in [7.

Lemma 2.2. a) $\mathbf{A}=\left(A_{1}, A_{2}\right)$ is hyponormal if and only if

$$
\left|\left(\left[A_{1}^{*}, A_{2}\right] x, y\right)\right|^{2} \leq\left(\left[A_{1}^{*}, A_{1}\right] x, x\right)\left(\left[A_{2}^{*}, A_{2}\right] y, y\right) \quad \text { for all } x, y \in \mathcal{H} .
$$

b) $\mathbf{A}=\left(A_{1}, A_{2}\right)$ is weakly hyponormal if and only if

$$
\left|\left(\left[A_{1}^{*}, A_{2}\right] x, x\right)\right|^{2} \leq\left(\left[A_{1}^{*}, A_{1}\right] x, x\right)\left(\left[A_{2}^{*}, A_{2}\right] x, x\right) \quad \text { for all } x \in \mathcal{H} .
$$

Let $U$ be the unilateral shift on $H^{2}$.

Proposition 2.3 (Farenick and McEachin, 1995). If $\left(U^{n}, T\right)$ is hyponormal, then $U^{* n} T U^{n}=T$. Therefore $T$ is a block Toeplitz operator with block size $n$. In particular, if $(U, T)$ is hyponormal, then $T$ is a Toeplitz operator.

Proof. If $\left(U^{n}, T\right)$ is hyponormal, then

$$
\begin{aligned}
& \left(\begin{array}{cc}
U^{* n} & 0 \\
0 & U^{n}
\end{array}\right)\left(\begin{array}{cc}
{\left[U^{* n}, U^{n}\right]} & {\left[T^{*}, U^{n}\right]} \\
{\left[U^{* n}, T\right]} & {\left[T^{*}, T\right]}
\end{array}\right)\left(\begin{array}{cc}
U^{n} & 0 \\
0 & U^{* n}
\end{array}\right) \\
& =\left(\begin{array}{cc}
U^{* n}\left[U^{* n}, U^{n}\right] U^{n} & U^{* n}\left[T^{*}, U^{n}\right] U^{* n} \\
U^{n}\left[U^{* n}, T\right] U^{n} & U^{n}\left[T^{*}, T\right] U^{* n}
\end{array}\right) \\
& =\left(\begin{array}{cc}
0 & U^{* n}\left[T^{*}, U^{n}\right] U^{* n} \\
U^{n}\left[U^{* n}, T\right] U^{n} & U^{n}\left[T^{*}, T\right] U^{* n}
\end{array}\right)
\end{aligned}
$$

is positive. Thus $U^{n}\left[U^{* n}, T\right] U^{n}=0$. Therefore

$$
U^{* n} U^{n}\left[U^{* n}, T\right] U^{n}=U^{* n} T U^{n}-T=0 .
$$

This completes the proof.

The above proposition is still valid if we replace $U$ by any isometry. The following proposition shows that if the other operator $T$ is also an isometry, conditions of this type are indeed sufficient for hyponormality.

Proposition 2.4. Let $S_{1}$ and $S_{2}$ be two isometries on $H^{2}$. Then $\mathbf{S}=\left(S_{1}, S_{2}\right)$ is hyponormal if and only if

$$
S_{1}=S_{2}^{*} S_{1} S_{2}, \quad S_{2}=S_{1}^{*} S_{2} S_{1} .
$$


Proof. We need to show the sufficiency. Let $H_{1}$ and $H_{2}$ be the ranges of $S_{1}$ and $S_{2}$ respectively. Let $P_{H_{1}}$ and $P_{H_{2}}$ be the projections onto $H_{1}$ and $H_{2}$. First note that

$$
\operatorname{Ker}\left(S_{1}^{*} S_{2}-S_{2} S_{1}^{*}\right) \supset \operatorname{Ker}\left(I-P_{H_{1}}\right)=H_{1} .
$$

Since, by (1), for any $h \in H^{2}$

$$
\left(S_{1}^{*} S_{2}-S_{2} S_{1}^{*}\right) S_{1} h=S_{1}^{*} S_{2} S_{1} h-S_{2} h=0,
$$

thus by Lemma 2.1

is positive if

$$
\left[\mathbf{S}^{*}, \mathbf{S}\right]=\left(\begin{array}{cc}
I-P_{H_{1}} & S_{2}^{*} S_{1}-S_{1} S_{2}^{*} \\
S_{1}^{*} S_{2}-S_{2} S_{1}^{*} & I-P_{H_{2}}
\end{array}\right)
$$

$$
I-P_{H_{2}} \geq\left(S_{1}^{*} S_{2}-S_{2} S_{1}^{*}\right)\left(I-P_{H_{1}}\right)\left(S_{2}^{*} S_{1}-S_{1} S_{2}^{*}\right) .
$$

Since $S_{1}^{*}\left(I-P_{H_{1}}\right)=0$ and $\left(I-P_{H_{1}}\right) S_{1}=0$, the above inequality is the same as

$$
I-P_{H_{2}} \geq S_{1}^{*} S_{2}\left(I-P_{H_{1}}\right) S_{2}^{*} S_{1} \text {. }
$$

Since $S_{1}^{*}$ and $S_{2}^{*}$ are contractions, the above inequality holds if and only if

$$
\left(I-P_{H_{1}}\right) S_{2}^{*} S_{1} P_{H_{2}}=\left(I-S_{1} S_{1}^{*}\right) S_{2}^{*} S_{1} S_{2} S_{2}^{*}=0 .
$$

Equivalently,

$$
S_{2}^{*} S_{1} S_{2} S_{2}^{*}=S_{1} S_{1}^{*} S_{2}^{*} S_{1} S_{2} S_{2}^{*}
$$

By (11), we see that the left side in the above equation is $S_{1} S_{2}^{*}$ and the right side is

$$
S_{1} S_{1}^{*} S_{2}^{*} S_{1} S_{2} S_{2}^{*}=S_{1} S_{2}^{*} S_{2} S_{2}^{*}=S_{1} S_{2}^{*} .
$$

This completes the proof.

\section{WEAKLY HYPONORMAL TOEPLITZ OPERATORS}

In this section we recall Cowen's characterization of hyponormal Toeplitz operators and prove a corollary. We give a necessary and sufficient condition for weak hyponormality for a class of Toeplitz pairs with rational symbols. We then give an example of a weakly hyponormal but not hyponormal Toeplitz pair.

The Hankel operator $H_{\psi}$ with symbol $\psi \in L^{\infty}$ is the operator on $H^{2}$ defined by

$$
H_{\psi} h=J(I-P)(\psi h),
$$

where $J$ is the unitary operator from $L^{2} \ominus H^{2}$ onto $H^{2}$ defined by $J\left(e^{-i n \theta}\right)=$ $e^{i(n-1) \theta}$ for $n \geq 1$. Toeplitz operators and Hankel operators are connected by the following important relation:

$$
T_{\phi \psi}-T_{\phi} T_{\psi}=H_{\phi}^{*} H_{\psi} .
$$

For $f \in L^{\infty}$, let $f_{+}=P(f)$ and $f_{-}=\overline{(I-P)(f)}$. Set

$$
\begin{gathered}
\psi=P(\psi)+(I-P)(\psi)=\psi_{+}+\overline{\psi_{-}}, \\
\phi=P(\phi)+(I-P)(\phi)=\phi_{+}+\overline{\phi_{-}} .
\end{gathered}
$$

The following formula, which expresses the self-commutator of $\mathbf{T}=\left(T_{\phi}, T_{\psi}\right)$ by Hankel operators, will play a crucial role in this paper:

$$
\left[\mathbf{T}^{*}, \mathbf{T}\right]=\left(\begin{array}{cc}
{\left[T_{\phi}^{*}, T_{\phi}\right]} & {\left[T_{\psi}^{*}, T_{\phi}\right]} \\
{\left[T_{\phi}^{*}, T_{\psi}\right]} & {\left[T_{\psi}^{*}, T_{\psi}\right]}
\end{array}\right)
$$




$$
=\left(\begin{array}{ll}
H_{\frac{*}{\phi_{+}}}^{*} H_{\overline{\phi_{+}}}-H_{\frac{*}{\phi_{-}}}^{*} H_{\overline{\phi_{-}}} & H_{\phi_{+}}^{*} H_{\overline{\psi_{+}}}-H_{\overline{\psi_{-}}}^{*} H_{\overline{\phi_{-}}} \\
H_{\frac{*}{\psi_{+}}}^{*} H_{\overline{\phi_{+}}}-H_{\frac{*}{\phi_{-}}}^{*} H_{\overline{\psi_{-}}} & H_{\frac{*}{\psi_{+}}}^{*} H_{\overline{\psi_{+}}}-H_{\frac{*}{\psi_{-}}} H_{\overline{\psi_{-}}}
\end{array}\right) .
$$

For $f \in L^{\infty}$, let $\tilde{f}=\overline{f(\bar{z})}$. We note that $H_{f}^{*}=H_{\tilde{f}}$.

Lemma 3.1. Let $\phi \in L^{\infty}, h \in H^{\infty}$ and $\theta \in H^{\infty}$ (an inner function). Then

$$
\begin{aligned}
H_{\phi} U & =U^{*} H_{\phi}, H_{\phi} T_{h}=H_{\phi h}=T_{\tilde{h}}^{*} H_{\phi}, \\
H_{\phi}^{*} H_{\phi}-H_{\theta \phi}^{*} H_{\theta \phi} & =H_{\phi}^{*} H_{\bar{\theta}} H_{\bar{\theta}}^{*} H_{\phi} .
\end{aligned}
$$

Proof. The first two equalities can be verified by the definition of a Hankel operator. By using the second equality and (2), we have

$$
H_{\phi}^{*} H_{\phi}-H_{\theta \phi}^{*} H_{\theta \phi}=H_{\phi}^{*}\left(I-T_{\tilde{\theta}} T_{\tilde{\theta}}^{*}\right) H_{\phi}=H_{\phi}^{*} H_{\tilde{\tilde{\theta}}}^{*} H_{\overline{\tilde{\theta}}} H_{\phi}=H_{\phi}^{*} H_{\bar{\theta}} H_{\bar{\theta}}^{*} H_{\phi} .
$$

This is the desired third equality in the lemma.

For an inner function $\theta \in H^{\infty}$, let $\mathcal{H}(\theta)=H^{2} \ominus \theta H^{2}$. The following lemma is well known.

Lemma 3.2. $\operatorname{Ker}\left(H_{f}\right) \neq\{0\}$ if and only if $f$ is of the form $\bar{\theta} b$, where $\theta$ is some inner function and $b \in H^{\infty}$ has the property that the inner part of $b$ and $\theta$ are coprime. Furthermore, we have

$$
\operatorname{Ker}\left(H_{\bar{\theta} b}\right)=\theta H^{2}, \quad \text { Closure }\left\{\operatorname{Range}\left(H_{\bar{\theta} b}\right)\right\}=\left\{\operatorname{Ker}\left(H_{\bar{\theta} b}^{*}\right)\right\}^{\perp}=\mathcal{H}(\tilde{\theta}) .
$$

The classical result of Kronecker on finite rank Hankel matrices is that $H_{f}$ is of finite rank if and only if $(I-P) f$ is a rational function, and in this case the rank is equal to the degree of $(I-P) f$. This can also be seen from the above lemma. The following result gives a way to compute the rank of a product of two Hankel operators.

Lemma 3.3 (Axler, Chang and Sarason, 1978). Let $f, g \in L^{\infty}$. Then

$$
\operatorname{Rank}\left(H_{f} H_{g}\right)=\min \left\{\operatorname{Rank}\left(H_{f}\right), \operatorname{Rank}\left(H_{g}\right)\right\} .
$$

In particular, $H_{f} H_{g}$ is of finite rank if and only if at least one of them is of finite rank.

We recall the following characterization of hyponormal Toeplitz operators.

Theorem 3.4 (Cowen, 1988). $T_{\phi}$ is hyponormal if and only if there exists a function $k \in H^{\infty}$ with $\|k\|_{\infty} \leq 1$ such that

$$
\overline{\phi_{-}}-k \overline{\phi_{+}} \in H^{2} \text {. }
$$

A slight variation of the above condition in [22] is that

$$
\phi-k \bar{\phi} \in H^{\infty},
$$

which only deals with bounded functions. A function $f$ is of bounded type if $f$ can be written as a quotient of two bounded analytic functions. A function of bounded type is of the form $\overline{q_{1}} q_{2} f_{0}$ for some inner functions $q_{1}, q_{2}$ and an outer function $f_{0}$. By Lemma 3.2 it is interesting to note that a bounded function $f$ is of bounded type if and only if $\operatorname{Ker}\left(H_{f}\right) \neq\{0\}$. It was observed in Lemma 6 [1] that if $T_{\phi}$ is hyponormal and if $\phi$ is not in $H^{\infty}$, then $\phi$ is of bounded type if and only if $\bar{\phi}$ is of bounded type. Assume this is the case. The positivity of

$$
\left[T_{\phi}^{*}, T_{\phi}\right]=H_{\overline{\phi_{+}}}^{*} H_{\overline{\phi_{+}}}-H_{\bar{\phi}_{-}}^{*} H_{\overline{\phi_{-}}}
$$


implies that

$$
\operatorname{Ker}\left(H_{\bar{\phi}_{+}}^{*} H_{\overline{\phi_{+}}}\right) \subset \operatorname{Ker}\left(H_{\bar{\phi}_{-}}^{*} H_{\overline{\phi_{-}}}\right) .
$$

By Lemma 3.2 we can write

$$
\phi_{+}=\theta_{1} \theta_{0} \bar{a}, \quad \phi_{-}=\theta_{1} \bar{b}
$$

for some inner functions $\theta_{1}, \theta_{0}$ and $a \in \mathcal{H}\left(\theta_{1} \theta_{0}\right)$ and $b \in \mathcal{H}\left(\theta_{1}\right)$, where the inner part of $a$ and $\theta_{1} \theta_{0}$ are relatively prime and the inner part of $b$ and $\theta_{1}$ are relatively prime.

Corollary 3.5. Let $\phi=\phi_{+}+\overline{\phi_{-}} \in L^{\infty}$. Assume

$$
\phi_{+}=\theta_{1} \theta_{0} \bar{a}, \quad \phi_{-}=\theta_{1} \bar{b}
$$

where $\theta_{1}, \theta_{0}$ are inner functions, and $a \in \mathcal{H}\left(\theta_{1} \theta_{0}\right)$ and $b \in \mathcal{H}\left(\theta_{1}\right)$. Let $\theta$ be a factor of $\theta_{0}$. Then $T_{\phi}$ is hyponormal if and only if $T_{\phi_{\theta}}$ is hyponormal, where

$$
\phi_{\theta}=P\left(\bar{\theta} \phi_{+}\right)+\overline{\phi_{-}} .
$$

Furthermore, if $\left[T_{\phi}^{*}, T_{\phi}\right]$ is of finite rank, then

$$
\operatorname{Rank}\left(\left[T_{\phi}^{*}, T_{\phi}\right]\right)=\operatorname{Degree}(\theta)+\operatorname{Rank}\left(\left[T_{\phi_{\theta}}^{*}, T_{\phi_{\theta}}\right]\right) .
$$

Proof. Assume $T_{\phi}$ is hyponormal. By Cowen's theorem, there exists a function $k \in H^{\infty}$ such that $\|k\|_{\infty} \leq 1$ and

$$
\overline{\theta_{1}} b-k \overline{\theta_{1} \theta_{0}} a=h
$$

for some $h \in H^{2}$. Equivalently,

$$
k a=\theta_{0}\left(b-\theta_{1} h\right) .
$$

Since the inner part of $a$ and $\theta_{1} \theta_{0}$ are coprime, $\theta_{0}$ is a factor of $k$. Thus $k=k_{1} \theta_{0}$. Let $\theta$ be a factor of $\theta_{0}$ and write $\theta_{0}=\theta \theta_{2}$. The above equation implies that

$$
\overline{\theta_{1}} b-k_{2} \overline{\theta_{1} \theta_{2}} a=h,
$$

where $\left\|k_{2}\right\|_{\infty}=\left\|k_{1} \theta_{2}\right\|_{\infty} \leq 1$. By Cowen's theorem, this proves that $T_{\phi_{\theta}}$ is hyponormal. The other implication is also clear by the above argument.

We next prove the rank formula. By Theorem 10 in Nakazi and Takahashi [22, $\left[T_{\phi}^{*}, T_{\phi}\right]$ is of finite rank if and only if there exist a finite Blaschke product $k$ and $h \in H^{2}$ satisfying

$$
\overline{\phi_{-}}-k \overline{\phi_{+}}=h
$$

and $k$ can be chosen such that the degree of $k$ is $\operatorname{Rank}\left(\left[T_{\phi}^{*}, T_{\phi}\right]\right)$. As before, we have $k=k_{1} \theta_{0}, \theta_{0}=\theta \theta_{2}, k_{2}=k_{1} \theta_{2}=k / \theta$ and

$$
\overline{\theta_{1}} b-k_{2} \overline{\theta_{1} \theta_{2}} a=\overline{\phi_{-}}-k_{2} \overline{\bar{\theta} \phi_{+}}=h \text {. }
$$

We need to show that

$$
\operatorname{Degree}\left(k_{2}\right)=\operatorname{Rank}\left(\left[T_{\phi_{\theta}}^{*}, T_{\phi_{\theta}}\right]\right) .
$$

Although the degree of $k$ is chosen to be $\operatorname{Rank}\left(\left[T_{\phi}^{*}, T_{\phi}\right]\right)$, the degree of $k_{2}$ is not chosen a priori. Nevertheless, by (3) and Lemma 3.1

$$
\left[T_{\phi}^{*}, T_{\phi}\right]=H_{\phi_{+}}^{*} H_{\overline{\psi_{+}}}-H_{k \overline{\phi_{+}}}^{*} H_{k \overline{\phi_{+}}}=H_{\bar{\phi}_{+}}^{*} H_{\bar{k}} H_{\bar{k}}^{*} H_{\overline{\phi_{+}}} .
$$


It follows that

$$
\operatorname{Degree}(k)=\operatorname{Rank}\left(\left[T_{\phi}^{*}, T_{\phi}\right]\right)=\operatorname{Rank}\left(H_{\phi_{+}}^{*} H_{\bar{k}}\right) \leq \operatorname{Rank}\left(H_{\overline{\phi_{+}}}\right)=\operatorname{Degree}\left(\theta_{1} \theta_{0}\right) \text {. }
$$

Therefore Degree $\left(\theta_{1} \theta_{2}\right) \geq \operatorname{Degree}\left(k_{2}\right)$. Similarly,

$$
\left[T_{\phi_{\theta}}^{*}, T_{\phi_{\theta}}\right]=H_{\theta \overline{\phi_{+}}}^{*} H_{\overline{k_{2}}} H_{\overline{k_{2}}}^{*} H_{\theta \overline{\phi_{+}}} .
$$

By Lemma 3.3 we have

$$
\operatorname{Rank}\left(\left[T_{\phi_{\theta}}^{*}, T_{\phi_{\theta}}\right]\right)=\min \left\{\operatorname{Degree}\left(\theta_{1} \theta_{2}\right), \operatorname{Degree}\left(k_{2}\right)\right\}=\operatorname{Degree}\left(k_{2}\right) .
$$

This completes the proof.

It was observed by the author [19] that the hyponormality of $T_{\phi}$ with rational symbol $\phi$ can be reduced to a tangential Hermite-Fejér interpolation problem; see Foias and Frazho [18], in particular pp. 294-304, for an extensive treatment of such a problem. We next show how to reduce the weak hyponormality of $\mathbf{T}=\left(T_{\phi}, T_{\psi}\right)$ for a certain class of rational symbols $\phi$ and $\psi$ to a Hermite-Fejér interpolation problem.

We first recall the classical Hermite-Fejér interpolation problem.

Problem. Let $\left\{\alpha_{i}, 1 \leq i \leq n\right\}$ be $n$ distinct complex numbers inside the unit disk. Let $\left\{b_{i j}: 1 \leq i \leq n\right.$ and $\left.0 \leq j \leq n_{i}\right\}$ be a given set of complex numbers. Find necessary and sufficient conditions for the existence of an analytic function $f \in H^{\infty}$ with $\|f\|_{\infty} \leq 1$ satisfying

$$
f^{(j)}\left(\alpha_{i}\right)=b_{i j}, \quad 1 \leq i \leq n, 0 \leq j \leq n_{i}
$$

where $f^{(j)}\left(\alpha_{i}\right)$ is the $j$-th derivative evaluated at $\alpha_{i}$.

If $n=1$, this is the so-called Carathéodory interpolation problem. If $n_{i}=1$ for all $i$, this is the so-called Nevanlinna-Pick interpolation problem.

For $|\alpha|<1$, set

$$
m_{\alpha}=\frac{z-\alpha}{1-\bar{\alpha} z} .
$$

Lemma 3.6. Let $\alpha_{i}$ for $i=1, \cdots, n$ and $\beta_{j}$ for $j=1, \cdots, l$ be distinct complex numbers inside the unit disk. Let

$$
\begin{aligned}
\theta_{1} & =\prod_{i=1}^{n} m_{\alpha_{i}}^{m_{i}}, \quad \theta_{2}=\prod_{j=1}^{l} m_{\beta_{j}}^{k_{j}} \\
\phi_{+} & =\theta_{1} \bar{a}, \quad \phi_{-}=\theta_{1} \bar{b} ; \quad \psi_{+}=\theta_{2} \bar{c}, \quad \psi_{-}=\theta_{2} \bar{d},
\end{aligned}
$$

where $a, b \in \mathcal{H}\left(\theta_{1}\right), c, d \in \mathcal{H}\left(\theta_{2}\right)$. Let $k_{1}, k_{2}$ be bounded analytic functions satisfying

$$
a-k_{1} b=\theta_{1} h_{1}, \quad c-k_{2} d=\theta_{2} h_{2}
$$

for some $h_{1}, h_{2} \in H^{\infty}$. Then $\mathbf{T}=\left(T_{\phi}, T_{\psi}\right)$ is weakly hyponormal if and only if for all constant $|\delta|=1$, there exists $k \in H^{\infty}$ with $\|k\|_{\infty} \leq 1$ such that

$$
\begin{gathered}
k^{(m)}\left(\alpha_{i}\right)=k_{1}^{(m)}\left(\alpha_{i}\right), \quad 0 \leq m \leq m_{i}, i=1, \cdots, n, \\
k^{(k)}\left(\beta_{j}\right)=\delta k_{2}^{(k)}\left(\beta_{j}\right), \quad 0 \leq k \leq k_{j}, j=1, \cdots, l .
\end{gathered}
$$

Proof. $\mathbf{T}=\left(T_{\phi}, T_{\psi}\right)$ is weakly hyponormal if $T_{\alpha \phi+\beta \psi}$ is hyponormal for all $\alpha$ and $\beta$. By Cowen's theorem, this is equivalent to the existence of an analytic function $k$ with $\|k\|_{\infty} \leq 1$ satisfying

$$
\alpha \overline{\theta_{1}} b+\beta \overline{\theta_{2}} d-k\left(\bar{\alpha} \overline{\theta_{1}} a+\overline{\beta \theta_{2}} c\right)=h
$$


for some $h \in H^{2}$. Equivalently,

$$
\alpha \theta_{2} b+\beta \theta_{1} d-k\left(\bar{\alpha} \theta_{2} a+\bar{\beta} \theta_{1} c\right)=\theta_{1} \theta_{2} h .
$$

By taking an appropriate number of derivatives in the above equation and evaluating the resulting equation at $\alpha_{i}$ and $\beta_{j}$, we see that $k$ satisfies the above equation if and only if

$$
\begin{array}{ll}
k^{(m)}\left(\alpha_{i}\right)=\frac{\alpha}{\bar{\alpha}} k_{1}^{(m)}\left(\alpha_{i}\right), & 0 \leq m \leq m_{i}, i=1, \cdots, n, \\
k^{(m)}\left(\beta_{j}\right)=\frac{\beta}{\bar{\beta}} k_{2}^{(k)}\left(\beta_{j}\right), & 0 \leq k \leq k_{j}, j=1, \cdots, l,
\end{array}
$$

where $k_{1}$ are $k_{2}$ are defined by (4). Now setting $\delta=\bar{\alpha} \beta / \alpha \bar{\beta}$, we see that $|\delta|=1$ and $\bar{\alpha} k / \alpha$ satisfies the interpolation conditions as in the lemma.

We now give an example of $\mathbf{T}=\left(T_{\phi}, T_{\psi}\right)$ which is weakly hyponormal but not hyponormal. Farenick and MaEachin [17] gave an example of $(U, W)$ for a weighted shift $W$ which is weakly hyponormal but not hyponormal. See also [14] for an example of a commuting pair $\left(T_{1}, T_{2}\right)$ which is weakly hyponormal but not hyponormal.

Example 1. Let $a, b, c$ and $d$ be constants such that $|a|>|b|,|c|>|d|, 0<$ $|\alpha|<1$ and

$$
\phi=z \bar{a}+\bar{z} b, \quad \psi=\frac{z \bar{c}}{1-\bar{\alpha} z}+\frac{\bar{z} d}{1-\alpha \bar{z}} .
$$

We claim that $\mathbf{T}=\left(T_{\phi}, T_{\psi}\right)$ is weakly hyponormal if and only if

$$
|\alpha|(|a c|+|b d|) \geq(|a d|+|b c|) .
$$

To see this, by the previous lemma, for all $|\delta|=1$, we need to find a bounded analytic function $k$ with $\|k\|_{\infty} \leq 1$ satisfying

$$
k(0)=\frac{b}{a}, \quad k(\alpha)=\delta \frac{d}{c} .
$$

This is a Nevanlinna-Pick interpolation problem with two interpolation conditions. It is solvable if and only if

$$
\left(\begin{array}{cc}
1-|k(0)|^{2} & 1-\overline{k(0)} k(\alpha) \\
1-k(0) \overline{k(\alpha)} & \frac{1-|k(\alpha)|^{2}}{1-|\alpha|^{2}}
\end{array}\right)
$$

is positive. A straightforward computation of the determinant of the above matrix shows that this is equivalent to (5). For example, if $\alpha=1 / 2, a=c=1, b=d=1 / 4$, then $\mathbf{T}=\left(T_{\phi}, T_{\psi}\right)$ is weakly hyponormal. But $\left(T_{\phi}, T_{\psi}\right)$ is not hyponormal as long as none of $a, b, c, d$ is zero. This follows from some observations as in Proposition 6.4 below.

\section{One Coordinate analytic Toeplitz operator}

Theorem 4.1. Assume $\phi=\phi_{+} \in H^{\infty}$ and $\psi=\psi_{+}+\overline{\psi_{-}} \in L^{\infty}$, where $\psi_{+} \in H^{2}$ and $\psi_{-} \in z H^{2}$.

a) If $\operatorname{Ker}\left(H_{\bar{\phi}}\right)=\{0\}$, then $\left(T_{\phi}, T_{\psi}\right)$ is hyponormal if and only if $\psi \in H^{\infty}$.

b) If $\operatorname{Ker}\left(H_{\bar{\phi}}\right)=\theta H^{2}$ for some inner function $\theta$, then $\phi=\theta \bar{f}$ for some $f \in H^{\infty}$, and $\left(T_{\phi}, T_{\psi}\right)$ is hyponormal if and only if $T_{\psi_{\theta}}$ is hyponormal, where

$$
\psi_{\theta}=P\left(\bar{\theta} \psi_{+}\right)+\overline{\psi_{-}} .
$$


Furthermore, if $\theta$ is a finite Blaschke product, then

$$
\operatorname{Rank}\left(\left[\mathbf{T}^{*}, \mathbf{T}\right]\right)=\operatorname{Degree}(\theta)+\operatorname{Rank}\left(\left[T_{\psi_{\theta}}^{*}, T_{\psi_{\theta}}\right]\right) .
$$

Proof. By the assumption $\phi_{-}=0$ and (3), we have

$$
\left[\mathbf{T}^{*}, \mathbf{T}\right]=\left(\begin{array}{cc}
{\left[T_{\phi}^{*}, T_{\phi}\right]} & {\left[T_{\psi}^{*}, T_{\phi}\right]} \\
{\left[T_{\phi}^{*}, T_{\psi}\right]} & {\left[T_{\psi}^{*}, T_{\psi}\right]}
\end{array}\right)=\left(\begin{array}{cc}
H_{\overline{\phi_{+}}}^{*} H_{\overline{\phi_{+}}} & H_{\overline{\phi_{+}}}^{*} H_{\overline{\psi_{+}}} \\
H_{\overline{\psi_{+}}}^{*} H_{\overline{\phi_{+}}} & {\left[T_{\psi}^{*}, T_{\psi}\right]}
\end{array}\right) .
$$

Therefore by Lemma 2.1 $\mathbf{T}=\left(T_{\phi}, T_{\psi}\right)$ is hyponormal if and only if

$$
\left|\left(H_{\psi_{+}}^{*} H_{\overline{\phi_{+}}} x, y\right)\right|^{2} \leq\left(H_{\overline{\phi_{+}}}^{*} H_{\overline{\phi_{+}}} x, x\right)\left(\left[T_{\psi}^{*}, T_{\psi}\right] y, y\right)
$$

for all $x, y \in H^{2}$. Equivalently,

$$
\left|\left(h, H_{\overline{\psi_{+}}} y\right)\right|^{2} \leq(h, h)\left(\left[T_{\psi}^{*}, T_{\psi}\right] y, y\right)
$$

for all $h \in \operatorname{Range}\left(H_{\overline{\phi_{+}}}\right)$and $y \in H^{2}$, and hence for all $h$ in the closure of Range $\left(H_{\overline{\phi_{+}}}\right)$and $y \in H^{2}$. There are two cases.

a) If $\operatorname{Ker}\left(H_{\bar{\phi}}\right)=\{0\}$, then the closure of $\operatorname{Range}\left(H_{\overline{\phi_{+}}}\right)$is $H^{2}$. Thus

$$
\left|\left(h, H_{\overline{\psi_{+}}} y\right)\right|^{2} \leq(h, h)\left(\left[T_{\psi}^{*}, T_{\psi}\right] y, y\right)
$$

for all $h \in H^{2}$. Let $h=H_{\overline{\psi_{+}}} y$; we have

$$
\left(H_{\overline{\psi_{+}}} y, H_{\overline{\psi_{+}}} y\right) \leq\left(H_{\overline{\psi_{+}}} y, H_{\overline{\psi_{+}}} y\right)-\left(H_{\overline{\psi_{-}}}, H_{\overline{\psi_{-}}} y\right)
$$

for all $y \in H^{2}$. Therefore $H_{\overline{\psi_{-}}}=0$, and so $\psi_{-}=0$.

b) If $\operatorname{Ker}\left(H_{\bar{\phi}}\right)=\theta H^{2}$ for some inner function $\theta$, the closure of $\operatorname{Range}\left(H_{\overline{\phi_{+}}}\right)$is $\mathcal{H}(\tilde{\theta})$, which is the same as Range $\left(H_{\bar{\theta}}\right)$. Equation (7) is the same as

$$
\left|\left(H_{\bar{\theta}} x, H_{\overline{\psi_{+}}} y\right)\right|^{2} \leq\left(H_{\bar{\theta}} x, H_{\bar{\theta}} x\right)\left(\left[T_{\psi}^{*}, T_{\psi}\right] y, y\right)
$$

for all $x, y \in H^{2}$. Note that $H_{\bar{\theta}} H_{\bar{\theta}}^{*}$ is the projection onto $\mathcal{H}(\tilde{\theta})$. By part c) of Lemma 2.1, the above equation is the same as

$$
\left[T_{\psi}^{*}, T_{\psi}\right]-H_{\overline{\psi_{+}}}^{*} H_{\bar{\theta}} H_{\bar{\theta}}^{*} H_{\overline{\psi_{+}}} \geq 0 .
$$

A straightforward computation shows that

$$
\begin{aligned}
{\left[T_{\psi_{\theta}}^{*}, T_{\psi_{\theta}}\right] } & =H_{\theta \overline{\psi_{+}}}^{*} H_{\theta \overline{\psi_{+}}}-H_{\overline{\psi_{-}}}^{*} H_{\overline{\psi_{-}}} \\
& =H_{\overline{\psi_{+}}}^{*} H_{\overline{\psi_{+}}}-H_{\overline{\psi_{-}}}^{*} H_{\overline{\psi_{-}}}-\left(H_{\overline{\psi_{+}}}^{*} H_{\overline{\psi_{+}}}-H_{\theta \overline{\psi_{+}}}^{*} H_{\theta \overline{\psi_{+}}}\right) \\
& =\left[T_{\psi}^{*}, T_{\psi}\right]-H_{\overline{\psi_{+}}}^{*} H_{\bar{\theta}} H_{\bar{\theta}}^{*} H_{\overline{\psi_{+}}}
\end{aligned}
$$

where the last equality follows from Lemma 3.1 .

If $\theta$ is a finite Blaschke product, by a classical result of Kronecker, $H_{\bar{\theta} f}$ is of finite rank and the rank is equal to the degree of $\theta$. We note that in this case $H_{\bar{\phi}}\left(H_{\bar{\phi}}^{*} H_{\bar{\phi}}\right)^{\#} H_{\bar{\phi}}^{*}$ is the projection $H_{\bar{\theta}} H_{\bar{\theta}}^{*}$ on $\mathcal{H}(\tilde{\theta})$, and

$$
\left[T_{\psi}^{*}, T_{\psi}\right]-H_{\overline{\psi_{+}}}^{*} H_{\bar{\phi}}\left(H_{\bar{\phi}}^{*} H_{\bar{\phi}}\right)^{\#} H_{\bar{\phi}}^{*} H_{\overline{\psi_{+}}}=\left[T_{\psi}^{*}, T_{\psi}\right]-H_{\bar{\psi}_{+}}^{*} H_{\bar{\theta}} H_{\bar{\theta}}^{*} H_{\overline{\psi_{+}}}=\left[T_{\psi_{\theta}}^{*}, T_{\psi_{\theta}}\right] .
$$

By part d) of Lemma 2.1 and (6), we have

$$
\operatorname{Rank}\left(\left[\mathbf{T}^{*}, \mathbf{T}\right]\right)=\operatorname{Degree}(\theta)+\operatorname{Rank}\left(\left[T_{\psi_{\theta}}^{*}, T_{\psi_{\theta}}\right]\right) .
$$

This completes the proof. 
Remark 1 . When $\phi=z$, the above theorem without the rank formula is due to Farenick and McEachin [17]. When $\phi$ is an analytic polynomial, it is due to Curto and Lee [13], but the rank formula was only obtained there when $\psi$ is assumed to be a trigonometric polynomial.

Remark 2. The above theorem extends to block Toeplitz operators, and one consequence is a result conjectured by Curto and Lee 13. The hyponormality of block Toeplitz operators will be discussed in a future work.

Corollary 4.2. Assume $\phi=\phi_{+}=\theta \overline{a(z)} \in H^{\infty}$, where $\theta$ is inner and $a \in \mathcal{H}(\theta)$. Assume also that $\psi=\psi_{+}+\overline{\psi_{-}} \in L^{\infty}$ and

$$
\psi_{+}=\theta_{1} \theta_{0} \overline{c(z)}, \quad \psi_{-}=\theta_{1} \overline{d(z)},
$$

where $\theta_{1}$ and $\theta_{0}$ are inner, and $c(z) \in \mathcal{H}\left(\theta_{0} \theta_{1}\right)$ and $d(z) \in \mathcal{H}\left(\theta_{1}\right)$. If $\left(T_{\phi}, T_{\psi}\right)$ is hyponormal, then there exist inner functions $\Delta_{1}$ and $\Delta_{2}$ such that

$$
\bar{\theta} \theta_{1} \theta_{0}=\theta_{1} \Delta_{1} \overline{\Delta_{2}},
$$

where $\theta_{1} \Delta_{1}$ and $\Delta_{2}$ are coprime. In other words, the greatest common divisor of $\theta$ and $\theta_{1} \theta_{0}$ is a factor of $\theta_{0}$.

Proof. By the previous theorem, the hyponormality of $\left(T_{\phi}, T_{\psi}\right)$ implies the hyponormality of $T_{\psi_{\theta}}$, where

$$
\psi_{\theta}=P\left(\bar{\theta} \psi_{+}\right)+\overline{\psi_{-}} .
$$

Thus $\theta_{1} H^{2}=\operatorname{Ker}\left(H_{\overline{\psi_{-}}}\right) \supset \operatorname{Ker}\left(H_{\theta \overline{\psi_{+}}}\right)$. Set

$$
\bar{\theta} \theta_{1} \theta_{0}=\Delta \overline{\Delta_{2}}
$$

where the inner functions $\Delta$ and $\Delta_{2}$ are coprime. It is clear that $\operatorname{Ker}\left(H_{\theta \overline{\psi_{+}}}\right)=$ $\Delta H^{2}$. Therefore $\Delta=\theta_{1} \Delta_{1}$. Now equation (8) becomes

$$
\bar{\theta} \theta_{1} \theta_{0}=\theta_{1} \Delta_{1} \overline{\Delta_{2}} \text {. }
$$

This completes the proof.

Theorem 4.3. Assume $\phi_{i} \in H^{\infty}$ for $i=1,2, \cdots, n$, and $\psi=\psi_{+}+\overline{\psi_{-}} \in L^{\infty}$, where $\psi_{+} \in H^{2}$ and $\psi_{-} \in z H^{2}$. Let $\theta_{i}(i=1,2, \cdots, n)$ be inner functions. Assume $\operatorname{Ker}\left(H_{\overline{\phi_{i}}}\right)=\theta_{i} H^{2}$. Then $\mathbf{T}=\left(T_{\phi_{1}}, \cdots, T_{\phi_{n}}, T_{\psi}\right)$ is hyponormal if and only if $T_{\psi_{\theta}}$ is hyponormal, where $\theta$ is the least common multiple of $\theta_{1}, \theta_{2}, \cdots, \theta_{n}$ and

$$
\psi_{\theta}=P\left(\bar{\theta} \psi_{+}\right)+\overline{\psi_{-}} .
$$

Moreover, if $\theta$ is a finite Blaschke product, then

$$
\operatorname{Rank}\left(\left[\mathbf{T}^{*}, \mathbf{T}\right]\right)=\operatorname{Degree}(\theta)+\operatorname{Rank}\left(\left[T_{\psi_{\theta}}^{*}, T_{\psi_{\theta}}\right]\right) .
$$

Proof. The proof is similar to the proof of Theorem 4.1, with suitable notational changes. Let

$$
\bigoplus_{i=1}^{n} H^{2}=H^{2} \oplus \cdots \oplus H^{2}
$$

be the direct sum of $n$ copies of $H^{2}$, and let

$$
\mathbf{H}_{\bar{\phi}}=\left(H_{\overline{\phi_{1}}}, \cdots, H_{\overline{\phi_{n}}}\right)
$$


be an operator from $\bigoplus_{i=1}^{n} H^{2}$ into $H^{2}$. By the assumption $\phi_{i} \in H^{\infty}$ and (B]), we have

$$
\left[\mathbf{T}^{*}, \mathbf{T}\right]=\left(\begin{array}{cc}
\mathbf{H}_{\bar{\phi}}^{*} \mathbf{H}_{\bar{\phi}} & \mathbf{H}_{\bar{\phi}}^{*} H_{\overline{\psi_{+}}} \\
H_{\overline{\psi_{+}}}^{*} \mathbf{H}_{\bar{\phi}} & {\left[T_{\psi}^{*}, T_{\psi}\right]}
\end{array}\right) .
$$

By Lemma 2.2 $\mathbf{T}$ is hyponormal if and only if

$$
\left|\left(H_{\psi_{+}}^{*} \mathbf{H}_{\bar{\phi}} x, y\right)\right|^{2} \leq\left(\mathbf{H}_{\bar{\phi}}^{*} \mathbf{H}_{\bar{\phi}} x, x\right)\left(\left[T_{\psi}^{*}, T_{\psi}\right] y, y\right)
$$

for all $x \in \bigoplus_{i=1}^{n} H^{2}$ and $y \in H^{2}$. Equivalently,

$$
\left|\left(h, H_{\overline{\psi_{+}}} y\right)\right|^{2} \leq(h, h)\left(\left[T_{\psi}^{*}, T_{\psi}\right] y, y\right)
$$

for all $h$ in the closure of $\operatorname{Range}\left(\mathbf{H}_{\bar{\phi}}\right)$ and $y \in H^{2}$. Note that

$$
\operatorname{Range}\left(\mathbf{H}_{\bar{\phi}}\right)=\left\{\sum_{i=1}^{n} h_{i}: h_{i} \in \operatorname{Range}\left(H_{\overline{\phi_{i}}}\right)\right\},
$$

and the closure of Range $\left(H_{\overline{\phi_{i}}}\right)$ is $\mathcal{H}\left(\tilde{\theta}_{i}\right)$. Therefore the closure of $\operatorname{Range}\left(\mathbf{H}_{\bar{\phi}}\right)$ is $\mathcal{H}(\tilde{\theta})$, where $\theta$ is the least common multiple of $\theta_{1}, \theta_{2}, \cdots, \theta_{n}$. Again note that $\mathcal{H}(\tilde{\theta})$ is the same as Range $\left(H_{\bar{\theta}}\right)$. Equation (9) now becomes

$$
\left|\left(H_{\bar{\theta}} x, H_{\overline{\psi_{+}}} y\right)\right|^{2} \leq\left(H_{\bar{\theta}} x, H_{\bar{\theta}} x\right)\left(\left[T_{\psi}^{*}, T_{\psi}\right] y, y\right)
$$

for all $x, y \in H^{2}$. The exact same argument as in the proof of Theorem 4.1 shows that the above inequality is equivalent to the hyponormality of $T_{\psi_{\theta}}$.

If $\theta$ is a finite Blaschke product, we note that $\mathbf{H}_{\bar{\phi}}\left(\mathbf{H}_{\bar{\phi}}^{*} \mathbf{H}_{\bar{\phi}}\right)^{\#} \mathbf{H}_{\bar{\phi}}{ }^{*}$ is the projection $H_{\bar{\theta}} H_{\bar{\theta}}^{*}$ on $\mathcal{H}(\tilde{\theta})$. The rank formula follows as in the proof of Theorem 4.1.

Corollary 4.4. Assume $\phi_{i} \in H^{\infty}$ and $\operatorname{Ker}\left(H_{\overline{\phi_{i}}}\right)=\theta_{i} H^{2}$, where $\theta_{i}(i=1,2, \cdots, n)$ are inner. Assume also

$$
\psi_{+}=\Delta \Delta_{0} \bar{c}, \quad \psi_{-}=\Delta \bar{d},
$$

where $\Delta$ and $\Delta_{0}$ are inner, $c \in \mathcal{H}\left(\Delta \Delta_{0}\right)$ and $d \in \mathcal{H}(\Delta)$. If $\theta_{i}$ is a factor of $\Delta_{0}$, then $\mathbf{T}=\left(T_{\phi_{1}}, \cdots, T_{\phi_{n}}, T_{\psi}\right)$ is hyponormal if and only if $\theta_{i}$ is a factor of $\Delta_{0}$. Moreover, if $\left[T_{\psi}^{*}, T_{\psi}\right]$ is of finite rank, then

$$
\operatorname{Rank}\left(\left[\mathbf{T}^{*}, \mathbf{T}\right]\right)=\operatorname{Rank}\left(\left[T_{\psi}^{*}, T_{\psi}\right]\right) .
$$

Proof. By the above theorem, the hyponormality of $\mathbf{T}$ is equivalent to the hyponormality of $T_{\psi_{\theta}}$, where $\theta$ is the least common multiple of $\theta_{1}, \cdots, \theta_{n}$. If $\mathbf{T}$ is hyponormal, then $\left(T_{\phi_{i}}, T_{\psi}\right)$ is hyponormal. By Corollary $4.2 \theta_{i}$ is a factor of $\Delta_{0}$, since by assumption $\theta_{i}$ is a factor of $\Delta_{0}$. Hence $\theta$ is a factor of $\Delta_{0}$. By Corollary 3.5, the hyponormality of $T_{\psi_{\theta}}$ is automatic by the hyponormality of $T_{\psi}$. The rank formula follows from the rank formulas in the above theorem and Corollary 3.5 This completes the proof.

Curto and Lee [13] conjectured that $\mathbf{T}=\left(T_{\phi_{1}}, \cdots, T_{\phi_{n}}\right)$ is hyponormal if and only if each subpair of $\mathbf{T}$ is hyponormal. The following example gives a negative answer to this conjecture.

Example 2. Let

$$
m_{\alpha}=\frac{z-\alpha}{1-\bar{\alpha} z}, \quad m_{\beta}=\frac{z-\beta}{1-\bar{\beta} z}
$$


where $\alpha$ and $\beta$ are two distinct nonzero complex numbers inside the unit disk. Let $\psi=z+\gamma \bar{z}$, where $\gamma$ is a complex number such that

$$
|\alpha \beta|<|\gamma|<\min \{|\alpha|,|\beta|\} .
$$

We claim that $\left(T_{m_{\alpha}}, T_{m_{\beta}}, T_{\psi}\right)$ is not hyponormal while both $\left(T_{m_{\alpha}}, T_{\psi}\right)$ and $\left(T_{m_{\beta}}, T_{\psi}\right)$ are hyponormal. Equivalently, by the above theorem, $T_{\psi_{m_{\alpha} m_{\beta}}}$ is not hyponormal while both $T_{\psi_{m_{\alpha}}}$ and $T_{\psi_{m_{\beta}}}$ are hyponormal.

To see this, note that

$$
\psi_{m_{\alpha}}=P\left(\overline{m_{\alpha}} z\right)+\gamma \bar{z}=1-|\alpha|^{2}-\bar{\alpha} z+\gamma \bar{z} .
$$

By the assumption that $|\alpha|>|\gamma|, T_{\psi_{m_{\alpha}}}$ is hyponormal. Similarly, by the assumption $|\beta|>|\gamma|, T_{\psi_{m_{\beta}}}$ is hyponormal. A simple computation shows that

$$
\psi_{m_{\alpha} m_{\beta}}=P\left(\overline{m_{\alpha} m_{\beta}} z\right)+\gamma \bar{z}=-(\bar{\alpha}+\bar{\beta})+(\alpha+\beta) \overline{\alpha \beta}+\overline{\alpha \beta} z+\gamma \bar{z} .
$$

By the assumption $|\alpha \beta|<|\gamma|, T_{\psi_{m_{\alpha} m_{\beta}}}$ is not hyponormal.

\section{TOEPLITZ OPERATORS WITH EQUAL ANTI-ANALYTIC PARTS}

The following simple lemma turns out to be very useful for studying the hyponormality of Toeplitz operators.

Lemma 5.1. (1) Let $\mathbf{T}=\left(T_{\phi_{1}}, \cdots, T_{\phi_{n}}, T_{\psi}\right)$ and $\mathbf{S}=\left(T_{\phi_{1}-\beta_{1} \psi}, \cdots, T_{\phi_{n}-\beta_{n} \psi}, T_{\psi}\right)$, where $\beta_{1}, \cdots, \beta_{n}$ are constants. $\mathbf{T}$ is hyponormal if and only if $\mathbf{S}$ is hyponormal. Furthermore,

$$
\operatorname{Rank}\left(\left[\mathbf{S}^{*}, \mathbf{S}\right]\right)=\operatorname{Rank}\left(\left[\mathbf{T}^{*}, \mathbf{T}\right]\right) .
$$

(2) Let $\mathbf{T}=\left(T_{\phi_{1}}, \cdots, T_{\phi_{n}}\right)$ and $\mathbf{S}=\left(T_{\beta_{1} \phi_{1}}, \cdots, T_{\beta_{n} \phi_{1}}\right)$, where $\beta_{1}, \cdots, \beta_{n}$ are constants and no $\beta_{i}$ is zero. $\mathbf{T}$ is hyponormal if and only if $\mathbf{S}$ is hyponormal. Furthermore,

$$
\operatorname{Rank}\left(\left[\mathbf{S}^{*}, \mathbf{S}\right]\right)=\operatorname{Rank}\left(\left[\mathbf{T}^{*}, \mathbf{T}\right]\right) .
$$

Proof. To prove (1), without loss of generality, assume $n=1$. Let $\mathbf{T}=\left(T_{\phi}, T_{\psi}\right)$ and $\mathbf{S}=\left(T_{\phi-\beta \psi}, T_{\psi}\right)$. A direct computation shows that

$$
\begin{aligned}
{\left[\mathbf{S}^{*}, \mathbf{S}\right] } & =\left(\begin{array}{cc}
{\left[T_{\phi-\beta \psi}^{*}, T_{\phi-\beta \psi}\right]} & {\left[T_{\psi}^{*}, T_{\phi-\beta \psi}\right]} \\
{\left[T_{\phi-\beta \psi}^{*}, T_{\psi}\right]} & {\left[T_{\psi}^{*}, T_{\psi}\right]}
\end{array}\right) \\
& =\left(\begin{array}{cc}
I & -\beta I \\
0 & I
\end{array}\right)\left(\begin{array}{cc}
{\left[T_{\phi}^{*}, T_{\phi}\right]} & {\left[T_{\psi}^{*}, T_{\phi}\right]} \\
{\left[T_{\phi}^{*}, T_{\psi}\right]} & {\left[T_{\psi}^{*}, T_{\psi}\right]}
\end{array}\right)\left(\begin{array}{cc}
I & 0 \\
-\bar{\beta} I & I
\end{array}\right) .
\end{aligned}
$$

The result follows from the above relation.

Statement (2) follows immediately from the fact that

$$
\begin{aligned}
{\left[\mathbf{S}^{*}, \mathbf{S}\right] } & =\left(\left[\overline{\beta_{j}} T_{\phi_{j}}^{*}, \beta_{i} T_{\phi_{i}}\right]\right)=\left(\overline{\beta_{j}} \beta_{i}\left[T_{\phi_{j}}^{*}, T_{\phi_{i}}\right]\right) \\
& =D\left(\left[T_{\phi_{j}}^{*}, T_{\phi_{i}}\right]\right) D^{*}=D\left[\mathbf{T}^{*}, \mathbf{T}\right] D^{*},
\end{aligned}
$$

where $D$ is the block diagonal operator with diagonal $\left(\beta_{1} I, \cdots, \beta_{n} I\right)$. 
Proposition 5.2. Assume $\phi_{-}=\alpha \psi_{-} \neq 0$ for some constant $\alpha$.

1. If $\phi$ or $\psi$ is not of bounded type, then $\mathbf{T}=\left(T_{\phi}, T_{\psi}\right)$ is hyponormal if and only if $\bar{\phi}-\bar{\alpha} \bar{\psi}$ is of bounded type.

2. If both $\phi$ and $\psi$ are of bounded type, we write

$$
\begin{gathered}
\phi_{+}=\theta \theta_{0} \theta_{1} \bar{a}, \quad \phi_{-}=\alpha \theta \bar{b}, \\
\psi_{+}=\theta \theta_{0} \theta_{2} \bar{c}, \quad \psi_{-}=\theta \bar{b},
\end{gathered}
$$

where $\theta_{1}$ and $\theta_{2}$ are coprime, $a \in \mathcal{H}\left(\theta \theta_{0} \theta_{1}\right), c \in \mathcal{H}\left(\theta \theta_{0} \theta_{2}\right)$ and $b \in \mathcal{H}(\theta)$. Then $\mathbf{T}=\left(T_{\phi}, T_{\psi}\right)$ is hyponormal if and only if

$$
\alpha P_{\mathcal{H}(\theta)}\left(\theta_{1} c\right)=P_{\mathcal{H}(\theta)}\left(\theta_{2} a\right),
$$

$\theta_{1}$ and $\theta$ are coprime, $\theta_{2}$ and $\theta$ are coprime, and both $T_{\psi_{\theta_{0} \theta_{1} \theta_{2}}}$ and $T_{\phi_{\theta_{0} \theta_{1} \theta_{2}}}$ are hyponormal, where

$$
\begin{aligned}
& \psi_{\theta_{0} \theta_{1} \theta_{2}}=P\left(\overline{\theta_{0} \theta_{1} \theta_{2}} \psi_{+}\right)+\overline{\psi_{-}}=P\left(\overline{\theta_{1}} \theta \bar{c}\right)+\bar{\theta} b, \\
& \phi_{\theta_{0} \theta_{1} \theta_{2}}=P\left(\overline{\theta_{0} \theta_{1} \theta_{2}} \phi_{+}\right)+\overline{\phi_{-}}=P\left(\overline{\theta_{2}} \theta \bar{a}\right)+\bar{\alpha} \bar{\theta} b .
\end{aligned}
$$

Proof. By the previous lemma, we can assume $\alpha=1$. We first prove the necessity part of $(1)$. Assume $\left(T_{\phi}, T_{\psi}\right)$ is hyponormal. By Lemma 5.1, $\left(T_{\phi-\psi}, T_{\psi}\right)$ is hyponormal. By the assumption $\phi_{-}=\psi_{-}, \phi-\psi=\phi_{+}-\psi_{+} \in H^{\infty}$. Thus by Theorem 4.1. $\operatorname{Ker} H_{\bar{\phi}-\bar{\psi}}=\theta H^{2} \neq\{0\}$ for some inner function $\theta$. Therefore $\bar{\phi}-\bar{\psi}=\bar{\theta} h$ for some $h \in \mathcal{H}(\theta)$. Hence $\bar{\phi}-\bar{\psi}$ is of bounded type.

Next we prove the sufficiency part of (1). Without loss of generality assume $\phi$ is not of bounded type. Assume that

$$
\bar{\phi}-\bar{\psi}=\overline{\phi_{+}}-\overline{\psi_{+}}=\bar{\theta} h,
$$

where $\theta$ is an inner function and $h \in \mathcal{H}(\theta) \cap H^{\infty}$. By Cowen's theorem, there exist $k_{1}, k_{2}, h_{1}, h_{2} \in H^{\infty}$ such that $\left\|k_{1}\right\|_{\infty} \leq 1,\left\|k_{2}\right\|_{\infty} \leq 1$ and

$$
\begin{aligned}
& \phi-k_{1} \bar{\phi}=h_{1}, \\
& \psi-k_{2} \bar{\psi}=h_{2} .
\end{aligned}
$$

Substituting (11) into (13), subtracting (12) from the resulting equation and multiplying by $\theta$, we have

$$
\theta\left(k_{2}-k_{1}\right) \bar{\phi}=\theta\left(h_{1}-h_{2}-\theta \bar{h}\right)+k_{2} h .
$$

If $k_{1} \neq k_{2}$, then $\bar{\phi}$ is of bounded type, which is a contradiction. Therefore $k_{1}=k_{2}$. It follows that $k_{2}=\theta k_{3}$ for some $k_{3} \in H^{\infty}$. Now equation (13) reads

$$
\psi-k_{3} \theta \bar{\psi}=h_{2} \text {. }
$$

By Cowen's theorem, this implies that $T_{\psi_{\theta}}$ is hyponormal, where

$$
\psi_{\theta}=P\left(\bar{\theta} \psi_{+}\right)+\overline{\psi_{-}} .
$$

By Theorem 4.1. $\left(T_{\phi-\psi}, T_{\psi}\right)$ is hyponormal. This concludes the proof of part (1).

Now we prove the necessity part of $(2)$. Assume $\left(T_{\phi}, T_{\psi}\right)$ is hyponormal. By Lemma $5.1\left(T_{\phi-\psi}, T_{\psi}\right)$ is hyponormal. Note that by our assumption $\phi-\psi \in H^{\infty}$. Therefore we can use Theorem 4.1 to study the hyponormality of $\left(T_{\phi-\psi}, T_{\psi}\right)$. We write

$$
\begin{aligned}
\phi-\psi & =\phi_{+}-\psi_{+}=\theta \theta_{0} \theta_{1} \bar{a}-\theta \theta_{0} \theta_{2} \bar{c} \\
& =\theta \theta_{0} \theta_{1} \theta_{2}\left[\overline{\theta_{2}} \bar{a}-\overline{\theta_{1}} \bar{c}\right] .
\end{aligned}
$$


Let

$$
\theta_{2} a-\theta_{1} c=\Delta_{0} \Delta_{1} e
$$

be the inner-outer factorization, where $e$ is outer and $\Delta_{0} \Delta_{1}$ is inner such that $\Delta_{0}$ is a factor of $\theta \theta_{0} \theta_{1} \theta_{2}$, and $\Delta_{1}$ and $\theta \theta_{0} \theta_{1} \theta_{2}$ are coprime. We claim that $\Delta_{0}$ and $\theta_{1} \theta_{2}$ are coprime. Equation (15) shows that a common inner factor of $\Delta_{0} \Delta_{1}$ and $\theta_{1}$ will be an inner factor of $\theta_{2} a$, which is impossible, since $\theta_{2}$ and $\theta_{1}$ are coprime and the inner part of $a$ and $\theta_{1}$ are also coprime. Similarly, $\theta_{2}$ and $\Delta_{0}$ are coprime. So in fact $\Delta_{0}$ is a factor of $\theta \theta_{0}$.

We next show that $\theta$ is a factor of $\Delta_{0}$. Set

$$
\Delta=\theta \theta_{0} \theta_{1} \theta_{2} \overline{\Delta_{0}}
$$

With the above notation, equation (14) becomes

$$
\phi_{+}-\psi_{+}=\Delta \overline{\Delta_{1}} \bar{e} .
$$

Therefore by Theorem 4.1, $T_{\psi_{\Delta}}$ is hyponormal, where

$$
\psi_{\Delta}=P\left(\bar{\Delta} \psi_{+}\right)+\overline{\psi_{-}} .
$$

In particular, by Corollary 4.2, this implies that

$$
\bar{\Delta} \theta \theta_{0} \theta_{2}=\theta \omega_{1} \overline{\omega_{2}}
$$

for some inner functions $\omega_{1}$ and $\omega_{2}$ such that $\theta \omega_{1}$ and $\omega_{2}$ are coprime. Substituting (16) into (17) and multiplying both sides of the equation by appropriate inner functions, we have

$$
\Delta_{0} \omega_{2}=\theta \omega_{1} \theta_{1} .
$$

Since $\omega_{2}$ and $\theta \omega_{1}$ are coprime and $\Delta_{0}$, and $\theta_{1}$ are coprime, we have $\Delta_{0}=\theta \omega_{1}$ and $\omega_{2}=\theta_{1}$. Therefore $\theta$ is a factor of $\Delta_{0}$, and $\theta_{1}$ and $\theta$ are coprime.

Now equation (15) becomes

$$
\theta_{2} a-\theta_{1} c=\theta \omega_{1} \Delta_{1} e .
$$

Therefore condition (10) holds. Note that $\omega_{1}$ is a factor of $\theta_{0}$, since $\Delta_{0}=\theta \omega_{1}$ is a factor of $\theta \theta_{0}$. We next show that the hyponormality of $T_{\psi_{\Delta}}$ implies the hyponormality of $T_{\psi_{\theta_{0} \theta_{1} \theta_{2}}}$. By Cowen's theorem, the hyponormality of $T_{\psi_{\Delta}}$ is equivalent to the existence of $k \in H^{\infty}$ and $h \in H^{2}$ such that $\|k\|_{\infty} \leq 1$ and

$$
\bar{\theta} b-k \Delta \overline{\theta \theta_{0} \theta_{2}} c=\bar{\theta} b-k \theta_{1} \overline{\theta \omega_{1}} c=h,
$$

where in the second equality we use (17) and $\omega_{2}=\theta_{1}$. Equivalently,

$$
\omega_{1} b-k \theta_{1} c=\omega_{1} \theta h .
$$

Note that the inner part of $c$ and $\omega_{1}$ is coprime, since $\omega_{1}$ is a factor of $\theta_{0}$. Also, as noted before, $\omega_{1}$ and $\theta_{1}$ are coprime. Therefore $k=k_{1} \omega_{1}$, where $k_{1} \in H^{\infty}$. The above equation becomes

$$
\bar{\theta} b-k_{1} \bar{\theta} \theta_{1} c=h .
$$

By Cowen's theorem again, this implies that $T_{\psi_{\theta_{0} \theta_{1} \theta_{2}}}$ is hyponormal. Similarly, $\theta_{2}$ and $\theta$ are coprime, and $T_{\phi_{\theta_{0} \theta_{1} \theta_{2}}}$ is hyponormal.

The proof of the sufficiency part of (2) is obtained by reversing the above argument and a direct application of Theorem 4.1 . 
Remark. It follows from the above proof that

$$
\operatorname{Ker}\left(H_{\overline{\phi_{+}}}-\overline{\psi_{+}}\right)=\Delta H^{2}=\theta \theta_{0} \theta_{1} \theta_{2} \overline{\Delta_{0}} H^{2} .
$$

Therefore by Lemma 5.1 and Theorem 4.1 we have

$$
\operatorname{Rank}\left(\left[\mathbf{T}^{*}, \mathbf{T}\right]\right)=\operatorname{Degree}(\Delta)+\operatorname{Rank}\left(\left[T_{\psi_{\Delta}}^{*}, T_{\psi_{\Delta}}\right]\right) .
$$

We also note that if condition (10) holds, then the hyponormality of $T_{\psi_{\theta_{0} \theta_{1} \theta_{2}}}$ implies the hyponormality of $T_{\phi_{\theta_{0} \theta_{1} \theta_{2}}}$, and vice versa.

Let $\phi_{i} \in L^{\infty}$ for $i=1,2, \cdots, n$ and $\psi \in L^{\infty}$.

Theorem 5.3. Assume $\left(\phi_{i}\right)_{-}=\psi_{-}$for $i=1,2, \cdots, n$.

1. If one of $\phi_{1}, \cdots, \phi_{n}, \psi$ is not of bounded type, then $\mathbf{T}=\left(T_{\phi_{1}}, \cdots, T_{\phi_{n}}, T_{\psi}\right)$ is hyponormal if and only if $\overline{\phi_{i}}-\bar{\psi}$ is of bounded type for $i=1, \cdots, n$.

2. If all $\phi_{1}, \cdots, \phi_{n}, \psi$ are of bounded type, we write

$$
\begin{aligned}
\left(\phi_{i}\right)_{+} & =\theta \Delta_{i} \theta_{i} \overline{a_{i}}, \quad\left(\phi_{i}\right)_{-}=\theta \bar{b}, \quad i=1,2, \cdots, n, \\
\psi_{+} & =\theta \Delta_{0} \bar{c}=\theta \Delta_{i} \delta_{i} \bar{c}, \quad \psi_{-}=\theta \bar{b},
\end{aligned}
$$

where $\theta_{i}$ and $\delta_{i}$ are coprime, $a_{i} \in \mathcal{H}\left(\theta \Delta_{i} \theta_{i}\right), c \in \mathcal{H}\left(\theta \Delta_{0}\right)$ and $b \in \mathcal{H}(\theta)$. Then $\mathbf{T}=\left(T_{\phi_{1}}, \cdots, T_{\phi_{n}}, T_{\psi}\right)$ is hyponormal if and only if

$$
P_{\mathcal{H}(\theta)}\left(\theta_{i} c\right)=P_{\mathcal{H}(\theta)}\left(\delta_{i} a_{i}\right), \quad i=1,2, \cdots, n,
$$

and $T_{\psi_{\theta_{0} \Delta_{0}}}$ is hyponormal, where $\theta_{0}$ is the least common multiple of $\theta_{1}, \theta_{2}, \cdots, \theta_{n}$ and

$$
\psi_{\theta_{0} \Delta_{0}}=P\left(\overline{\theta_{0} \Delta_{0}} \psi_{+}\right)+\overline{\psi_{-}} .
$$

Proof. We first prove part (1). The necessity clearly follows from the previous proposition. For the sufficiency, we write

$$
\overline{\phi_{i}}-\bar{\psi}=\overline{\theta_{i}} h_{i}, \quad i=1, \cdots, n,
$$

where $\theta_{i}$ is inner and $h_{i} \in \mathcal{H}\left(\theta_{i}\right)$. Let $k \in H^{\infty}, h \in H^{2}$ be such that $\|k\|_{\infty} \leq 1$ and

$$
\overline{\psi_{-}}-k \overline{\psi_{+}}=h \text {. }
$$

As in the proof of the previous proposition, $\theta_{i}$ is an inner factor of $k$. Therefore $\theta_{0}$, the least common multiple of $\theta_{1}, \cdots, \theta_{n}$, is an inner factor of $k$; that is, $k=$ $\theta_{0} k_{1}$. By Cowen's theorem, equation (19) implies the hyponormality of $T_{\psi_{\theta_{0}}}$. By Theorem 4.3, this implies the hyponormality of $\left(T_{\phi_{1}-\psi}, \cdots, T_{\phi_{n}-\psi}, T_{\psi}\right)$, hence the hyponormality of $\mathbf{T}=\left(T_{\phi_{1}}, \cdots, T_{\phi_{n}}, T_{\psi}\right)$.

We now show the sufficiency of part (2). Equation (18) implies that there exists some $h_{i} \in H^{2}$ such that

$$
\delta_{i} a_{i}-\theta_{i} c=\theta h_{i} .
$$

Note that the inner part of $h_{i}$ and $\delta_{i} \theta_{i}$ are coprime, since $\delta_{i}$ and $\theta_{i}$ are coprime. Thus

$$
\begin{aligned}
\phi_{i}-\psi & =\left(\phi_{i}\right)_{+}-\psi_{+}=\theta \Delta_{i} \theta_{i} \delta_{i}\left(\overline{\delta_{i} a_{i}}-\overline{\theta_{i} c}\right) \\
& =\theta \Delta_{i} \theta_{i} \delta_{i}\left(\overline{\theta h_{i}}\right)=\Delta_{i}^{\prime} \delta_{i} \theta_{i} \overline{h_{i}^{\prime}},
\end{aligned}
$$

where $\Delta_{i}^{\prime}$ is a factor of $\Delta_{i}$ and the inner part of $h_{i}^{\prime}$ and $\Delta_{i}^{\prime} \delta_{i} \theta_{i}$ are coprime. Let $\Delta$ be the least common multiple of the $\Delta_{i}^{\prime} \delta_{i} \theta_{i}, i=1, \cdots, n$. Note that $\Delta=\delta \theta_{0}$, where $\theta_{0}$ is the least common multiple of $\theta_{1}, \ldots, \theta_{n}$ and $\delta$ is a factor of $\Delta_{i} \delta_{i}=\Delta_{0}$. Similarly to the proof of the previous proposition, we can use Cowen's theorem to 
show that the hyponormality of $T_{\psi_{\theta_{0} \Delta_{0}}}$ implies the hyponormality of $T_{\psi_{\Delta}}\left(=T_{\psi_{\theta_{0} \delta}}\right)$. By Theorem 4.3 we have that $\left(T_{\phi_{1}-\psi}, \cdots, T_{\phi_{n}-\psi}, T_{\psi}\right)$ is hyponormal. Thus $\mathbf{T}=$ $\left(T_{\phi_{1}}, \cdots, T_{\phi_{n}}, T_{\psi}\right)$ is hyponormal.

Next we prove the necessity of part (2). The hyponormality of $\left(T_{\phi_{1}}, \cdots, T_{\phi_{n}}, T_{\psi}\right)$ implies the hyponormality of $\left(T_{\phi_{i}}, T_{\psi}\right)$ for each $i$. By the previous proposition, this shows that equation (18) holds. Now the arguments in the proof of sufficiency are reversible. This completes the proof.

Remark. It follows from the above proof that for $i=1, \cdots, n$,

$$
\operatorname{Ker}\left(H_{\overline{\phi_{i}}-\bar{\psi}}\right)=\Delta_{i}^{\prime} \delta_{i} \theta_{i} H^{2} \text {. }
$$

Therefore by Lemma 5.1 and Theorem 4.3 we have

$$
\operatorname{Rank}\left(\left[\mathbf{T}^{*}, \mathbf{T}\right]\right)=\operatorname{Degree}(\Delta)+\operatorname{Rank}\left(\left[T_{\psi_{\Delta}}^{*}, T_{\psi_{\Delta}}\right]\right),
$$

where $\Delta$ is the least common multiple of the $\Delta_{i}^{\prime} \delta_{i} \theta_{i}, i=1, \cdots, n$.

A similar result for the hyponormality of $\left(T_{\phi_{1}}, \cdots, T_{\phi_{l}}, T_{\phi_{l+1}}, \cdots, T_{\phi_{n}}, T_{\psi}\right)$ holds in the more general case in which $\phi_{i} \in H^{\infty}$ for $1 \leq i \leq l$ and $\left(\phi_{i}\right)_{-}=\psi_{-}$for $l+1 \leq i \leq n$. A special case of this result is the following corollary.

Corollary 5.4. Assume that

$$
\begin{aligned}
\phi_{i} & \in H^{\infty}, \quad \operatorname{Ker}\left(H_{\overline{\phi_{i}}}\right)=\Delta_{i} H^{2}, \quad i=1, \cdots, l, \\
\left(\phi_{i}\right)_{+} & =\theta \Delta_{i} \overline{a_{i}}, \quad\left(\phi_{i}\right)_{-}=\theta \bar{b}, \quad i=l+1, \cdots, n, \\
\psi_{+} & =\theta \Delta_{0} \bar{c}=\theta \Delta_{i} \delta_{i} \bar{c}, \quad \psi_{-}=\theta \bar{b},
\end{aligned}
$$

where $\Delta_{i}(i=1, \cdots, n)$ are factors of $\Delta_{0}, a_{i} \in \mathcal{H}\left(\theta \Delta_{i}\right), c \in \mathcal{H}\left(\theta \Delta_{0}\right)$ and $b \in \mathcal{H}(\theta)$. Then $\mathbf{T}=\left(T_{\phi_{1}}, \cdots, T_{\phi_{n}}, T_{\psi}\right)$ is hyponormal if and only if

$$
P_{\mathcal{H}(\theta)}(c)=P_{\mathcal{H}(\theta)}\left(\delta_{i} a_{i}\right), \quad i=l+1, \cdots, n .
$$

If this is the case, then

$$
\operatorname{Rank}\left(\left[\mathbf{T}^{*}, \mathbf{T}\right]\right)=\operatorname{Rank}\left(\left[T_{\psi}^{*}, T_{\psi}\right]\right) .
$$

Proof. By the proof of the previous theorem, $\mathbf{T}$ is hyponormal if 20 holds and $T_{\phi_{\theta_{0}} \Delta_{0}}$ is hyponormal for a certain inner function $\theta_{0}$. It follows from the assumption that $\theta_{0}=1$. Therefore by Corollary 3.5 , the hyponormality of $T_{\phi_{\Delta_{0}}}$ is guaranteed by that of $T_{\phi}$. The rank formula follows from the remark above and Corollary 3.5 .

\section{Miscellaneous Cases}

We begin with a general fact about the kernels of Hankel operators.

Lemma 6.1. Let $\theta$ be an inner function and $\psi \notin H^{2}$. If $\operatorname{Ker}\left(H_{\psi}^{*} H_{\phi}\right) \supset \theta H^{2}$, then $\operatorname{Ker}\left(H_{\phi}\right) \supset \theta H^{2}$. In particular, if $\operatorname{Ker}\left(H_{\psi}^{*} H_{\phi}\right) \supset \operatorname{Ker}\left(H_{\delta}\right)$, then $\operatorname{Ker}\left(H_{\phi}\right) \supset$ $\operatorname{Ker}\left(H_{\delta}\right)$.

Proof. The assumption implies that

$$
H_{\psi}^{*} H_{\phi} T_{\theta}=H_{\psi}^{*} H_{\phi \theta}=0 .
$$

By a result of Brown and Halmos [6], $H_{\phi \theta}=0$. Therefore $\operatorname{Ker}\left(H_{\phi}\right) \supset \theta H^{2}$.

Lemma 6.2. Assume neither $\phi$ nor $\psi$ is analytic. If $\left(T_{\phi}, T_{\psi}\right)$ is hyponormal, then $\operatorname{Ker}\left(H_{\overline{\phi_{+}}}\right) \subset \operatorname{Ker}\left(H_{\overline{\psi_{-}}}\right)$and $\operatorname{Ker}\left(H_{\overline{\psi_{+}}}\right) \subset \operatorname{Ker}\left(H_{\overline{\phi_{-}}}\right)$. 
Proof. First, note that the hyponormality of $T_{\phi}$ implies that

$$
\operatorname{Ker}\left(H_{\overline{\phi_{+}}}^{*} H_{\overline{\phi_{+}}}-H_{\overline{\phi_{-}}}^{*} H_{\overline{\phi_{-}}}\right) \supset \operatorname{Ker}\left(H_{\overline{\phi_{+}}}\right) .
$$

By equation (3) and Lemma 2.1, we have

$$
\operatorname{Ker}\left(H_{\overline{\psi_{+}}}^{*} H_{\overline{\phi_{+}}}-H_{\overline{\phi_{-}}}^{*} H_{\overline{\psi_{-}}}\right) \supset \operatorname{Ker}\left(H_{\overline{\phi_{+}}}^{*} H_{\overline{\phi_{+}}}-H_{\overline{\phi_{-}}}^{*} H_{\overline{\phi_{-}}}\right) \supset \operatorname{Ker}\left(H_{\overline{\phi_{+}}}\right) .
$$

Therefore $\operatorname{Ker}\left(H_{\overline{\phi_{-}}}^{*} H_{\overline{\psi_{-}}}\right) \supset \operatorname{Ker}\left(H_{\overline{\phi_{+}}}\right)$. By the previous lemma, $\operatorname{Ker}\left(H_{\overline{\psi_{-}}}\right) \supset$ $\operatorname{Ker}\left(H_{\overline{\phi_{+}}}\right)$. Similarly we can show that $\operatorname{Ker}\left(H_{\overline{\phi_{-}}}\right) \supset \operatorname{Ker}\left(H_{\overline{\psi_{+}}}\right)$.

Remark. The above observation shows that if $\left(T_{\phi}, T_{\psi}\right)$ is hyponormal, then $\phi$ is not of bounded type implies that $\psi$ is not of bounded type and vice versa.

The following two results follow from the above observation.

Proposition 6.3. Let $m_{\alpha}=(z-\alpha) /(1-\bar{\alpha} z)$,

$$
\begin{aligned}
& \phi_{+}=m_{\alpha} \theta_{0} \theta_{1} \bar{a}, \quad \phi_{-}=m_{\alpha} \theta_{0} \bar{b}, \\
& \psi_{+}=m_{\alpha} \theta_{2} \theta_{3} \bar{c}, \quad \psi_{-}=m_{\alpha} \theta_{2} \bar{d},
\end{aligned}
$$

where $a \in \mathcal{H}\left(m_{\alpha} \theta_{0} \theta_{1}\right), b \in \mathcal{H}\left(m_{\alpha} \theta_{0}\right), c \in \mathcal{H}\left(m_{\alpha} \theta_{2} \theta_{3}\right)$ and $d \in \mathcal{H}\left(m_{\alpha} \theta_{2}\right)$. Assume $m_{\alpha}$ and $\theta_{0} \theta_{1}$ are coprime, and $m_{\alpha}$ and $\theta_{2} \theta_{3}$ are coprime. Then $\left(T_{\phi}, T_{\psi}\right)$ is hyponormal if and only if $\phi=\beta \psi$, where

$$
\bar{\beta}=\frac{\theta_{2}(\alpha) \theta_{3}(\alpha) a(\alpha)}{\theta_{0}(\alpha) \theta_{1}(\alpha) c(\alpha)} .
$$

Proof. Let $\beta$ be defined as in (21). Note that

$$
\begin{aligned}
\phi_{+}-\beta \psi_{+} & =m_{\alpha} \theta_{0} \theta_{1}-\beta m_{\alpha} \theta_{2} \theta_{3} \\
& =\theta_{0} \theta_{1} \theta_{2} \theta_{3} m_{\alpha}\left(\overline{\theta_{2}} \overline{\theta_{3}} \bar{a}-\beta \overline{\theta_{0}} \overline{\theta_{1}} \bar{c}\right) .
\end{aligned}
$$

By the definition of $\beta$, we have $m_{\alpha}\left(\overline{\theta_{2}} \overline{\theta_{3}} \bar{a}-\beta \overline{\theta_{0}} \overline{\theta_{1}} \bar{c}\right) \in \overline{H^{2}}$. Thus

$$
\operatorname{Ker}\left(H_{\overline{\phi_{+}}-\beta \overline{\psi_{+}}}\right) \supset \theta_{0} \theta_{1} \theta_{2} \theta_{3} H^{2} .
$$

On the other hand, by Lemma 5.1, the hyponormality of $\left(T_{\phi}, T_{\psi}\right)$ implies the hyponormality of $\left(T_{\phi-\beta \psi}, T_{\psi}\right)$. Now by Lemma [6.2, we must have

$$
\theta_{0} \theta_{1} \theta_{2} \theta_{3} H^{2} \subset \operatorname{Ker}\left(H_{\overline{\phi_{+}}-\beta \overline{\psi_{+}}}\right) \subset \operatorname{Ker}\left(H_{\overline{\psi_{-}}}\right)=m_{\alpha} \theta_{2} H^{2} .
$$

This is a contradiction, unless $\phi=\beta \psi$.

Proposition 6.4. Assume that

$$
\begin{aligned}
& \phi_{+}=\theta_{0} \bar{a}, \quad \phi_{-}=\theta_{0} \bar{b}, \\
& \psi_{+}=\theta_{1} \bar{c}, \quad \psi_{-}=\theta_{1} \bar{d},
\end{aligned}
$$

where $\theta_{0}$ and $\theta_{1}$ are inner, $a, b \in \mathcal{H}\left(\theta_{0}\right)$ and $c, d \in \mathcal{H}\left(\theta_{1}\right)$. Then $\left(T_{\phi}, T_{\psi}\right)$ hyponormal implies that $\theta_{0}=\theta_{1}$. Furthermore, if $\theta_{0}$ is not a singular inner function, then $\phi=\beta \psi$ for some constant $\beta$. 
Proof. By Lemma 6.2

$$
\begin{aligned}
& \operatorname{Ker}\left(H_{\overline{\psi_{-}}}\right)=\theta_{1} H^{2} \supset \operatorname{Ker}\left(H_{\overline{\phi_{+}}}\right)=\theta_{0} H^{2}, \\
& \operatorname{Ker}\left(H_{\overline{\phi_{-}}}\right)=\theta_{0} H^{2} \supset \operatorname{Ker}\left(H_{\overline{\psi_{+}}}\right)=\theta_{1} H^{2} .
\end{aligned}
$$

Therefore $\theta_{1}=\theta_{0}$. If $\theta_{0}$ is not a singular inner function, say

$$
\theta_{0}=\theta_{1}=m_{\alpha} m_{\alpha}^{n} \Delta
$$

where $n \geq 0, m_{\alpha}$ and $\Delta$ are coprime and $m_{\alpha}=(z-\alpha) /(1-\bar{\alpha} z)$. Let $\beta=$ $\bar{a}(\alpha) / \bar{c}(\alpha)$. By Lemma 5.1, the hyponormality of $\left(T_{\phi}, T_{\psi}\right)$ implies the hyponormality of $\left(T_{\phi-\beta \psi}, T_{\psi}\right)$. By Lemma 6.2

$$
\operatorname{Ker}\left(H_{\overline{\psi_{-}}}\right)=m_{\alpha} m_{\alpha}^{n} H^{2} \supset \operatorname{Ker}\left(H_{(\phi-\beta \psi)_{+}}\right) .
$$

But $\operatorname{Ker}\left(H \overline{(\phi-\beta \psi)_{+}}\right) \supset m_{\alpha}^{n} \Delta H^{2}$, since

$$
(\phi-\beta \psi)_{+}=m_{\alpha}^{n} \Delta m_{\alpha}(\overline{a(z)}-\beta \overline{c(z)})
$$

and $\overline{m_{\alpha}}(a(z)-\bar{\beta} c(z)) \in H^{2}$. This is a contradiction, unless $\phi=\beta \psi$.

Recall that, for an inner function $\theta, \mathcal{H}(\theta)=H^{2} \ominus \theta H^{2}$. In particular, we have

$$
\mathcal{H}\left(z^{n}\right)=\{\text { all analytic polynomials of degress less than } n\} .
$$

Theorem 6.5. Assume that

$$
\begin{array}{ll}
\phi_{+}=z^{N} \theta_{0} \theta_{1} \overline{a(z)}, & \phi_{-}=z^{n} \overline{b(z)}, \\
\psi_{+}=z^{L} \theta_{0} \theta_{2} \overline{c(z)}, & \psi_{-}=z^{l} \overline{d(z)},
\end{array}
$$

where $a \in \mathcal{H}\left(z^{N} \theta_{0} \theta_{1}\right), b \in \mathcal{H}\left(z^{n}\right) c \in \mathcal{H}\left(z^{L} \theta_{0} \theta_{2}\right), d \in \mathcal{H}\left(z^{l}\right), \theta_{0}(0) \theta_{1}(0) \theta_{2}(0) \neq 0$ and $\theta_{1}$ and $\theta_{2}$ are coprime. Then $\mathbf{T}=\left(T_{\phi}, T_{\psi}\right)$ is hyponormal if and only if

$$
N=L, n=l, b(z)=\alpha d(z), \quad P_{\mathcal{H}\left(z^{n}\right)}\left[\theta_{2} a(z)\right]=\alpha P_{\mathcal{H}\left(z^{n}\right)}\left[\theta_{1} c(z)\right]
$$

for some constant $\alpha$ and $T_{\psi_{z^{N-n} \theta_{0} \theta_{1} \theta_{2}}}$ is hyponormal, where

$$
\psi_{z^{N-n} \theta_{0} \theta_{1} \theta_{2}}=P\left(\overline{z^{N-n} \theta_{0} \theta_{1} \theta_{2}} \psi_{+}\right)+\overline{\psi_{-}}=P\left(z^{l} \theta_{1} \bar{c}(z)\right)+\bar{z}^{l} d(z) .
$$

Proof. The sufficiency follows from Proposition 5.2 We prove the necessity. Without loss of generality, assume $l \geq n \geq 1$. We prove the theorem by induction on $n$. Assume $n=1$. If $l=1$, then $b(z)$ and $d(z)$ are nonzero constants $b$ and $d$. Therefore $b(z)=\alpha d(z)$ for $\alpha=b / d$. Now the remaining part of the theorem for $n=l=1$ follows from Proposition [5.2. For example, assume $L \geq N$; by (10) in Proposition 5.2, we have

$$
\alpha P_{\mathcal{H}(z)}\left(\theta_{1} c\right)=P_{\mathcal{H}(z)}\left(z^{L-N} \theta_{2} a\right) .
$$

Thus $L-N=0$, since otherwise $\alpha P_{\mathcal{H}(z)}\left(\theta_{1} c\right)=\alpha \theta_{1}(0) c(0)=0$, which is a contradiction. 
We now prove that $l=1$. If $l>1$, we first show that $L-N \geq l-1$. Note that the positivity of

$$
\begin{aligned}
& \left(\begin{array}{cc}
T_{z}^{*} & 0 \\
0 & T_{z}^{*}
\end{array}\right)[\mathbf{T}, \mathbf{T}]\left(\begin{array}{cc}
T_{z} & 0 \\
0 & T_{z}
\end{array}\right) \\
& =\left(\begin{array}{cc}
H_{z \overline{\phi_{+}}}^{*} H_{z \overline{\phi_{+}}}-H_{z \overline{\phi_{-}}}^{*} H_{z \overline{\phi_{-}}} & H_{z \overline{\phi_{+}}}^{*} H_{z \overline{\psi_{+}}}-H_{z \overline{\psi_{-}}}^{*} H_{z \overline{\phi_{-}}} \\
H_{z \overline{\psi_{+}}}^{*} H_{z \overline{\phi_{+}}}-H_{z \overline{\phi_{-}}}^{*} H_{z \overline{\psi_{-}}} & H_{z \overline{\psi_{+}}}^{*} H_{z \overline{\psi_{+}}}-H_{z \overline{\psi_{-}}}^{*} H_{z \overline{\psi_{-}}}
\end{array}\right) \\
& =\left(\begin{array}{cc}
H_{z \overline{\phi_{+}}}^{*} H_{z \overline{\phi_{+}}} & H_{z \overline{\phi_{+}}}^{*} H_{z \overline{\psi_{+}}} \\
H_{z \overline{\psi_{+}}}^{*} H_{z \overline{\phi_{+}}} & H_{z \overline{\psi_{+}}}^{*} H_{z \overline{\psi_{+}}}-H_{z \overline{\psi_{-}}}^{*} H_{z \overline{\psi_{-}}}
\end{array}\right)=\left[\mathbf{T}_{\mathbf{1}}, \mathbf{T}_{\mathbf{1}}\right]
\end{aligned}
$$

implies the hyponormality of $\mathbf{T}_{\mathbf{1}}=\left(T_{\phi_{1}}, T_{\psi_{1}}\right)$, where

$$
\phi_{1}=P\left(\bar{z} \phi_{+}\right), \quad \psi_{1}=P\left(\bar{z} \psi_{+}\right)+(I-P)\left(z \overline{\psi_{-}}\right) .
$$

Note that

$$
\begin{aligned}
& \bar{z} \psi_{-}=\bar{z} z^{l} \overline{d(z)}=z^{l-1} \overline{d_{1}(z)}+\beta, \\
& \bar{z} \psi_{+}=\bar{z} z^{L} \theta_{0} \theta_{2} \overline{c(z)}=z^{L-1} \theta_{0} \theta_{2} \overline{c_{1}(z)}+\gamma, \\
& \bar{z} \phi_{+}=\bar{z} z^{N} \theta_{0} \theta_{1} \overline{a(z)}=z^{N-1} \theta_{0} \theta_{1} \overline{a_{1}(z)}+\delta,
\end{aligned}
$$

where $d_{1}(z) \in \mathcal{H}\left(z^{l-1}\right), c_{1}(z) \in \mathcal{H}\left(z^{L-1} \theta_{0} \theta_{2}\right), a_{1}(z) \in \mathcal{H}\left(z^{N-1} \theta_{0} \theta_{1}\right)$, and $\beta, \gamma, \delta$ are constants such that

$$
\begin{aligned}
& d(z)=d_{1}(z)+z^{l-1} \bar{\beta}, \\
& c(z)=c_{1}(z)+z^{L-1} \theta_{0} \theta_{2} \bar{\gamma}, \\
& a(z)=a_{1}(z)+z^{N-1} \theta_{0} \theta_{1} \bar{\delta} .
\end{aligned}
$$

In the above we have used the fact that

$$
\mathcal{H}\left(z^{N} \theta_{0} \theta_{1}\right)=\mathcal{H}\left(z^{N-1} \theta_{0} \theta_{1}\right) \oplus z^{N-1} \theta_{0} \theta_{1} \mathcal{H}(z) .
$$

Since by the (implicit) assumption, $z^{L} \theta_{0} \theta_{2}$ and the inner part of $c(z)$ are relatively prime, it follows that $z^{L-1} \theta_{0} \theta_{2}$ and the inner part of $c_{1}(z)$ are also relatively prime. Set

$$
\begin{aligned}
& \psi_{2}=z^{L-1} \theta_{0} \theta_{2} \overline{c_{1}(z)}+\overline{z^{l-1} \overline{d_{1}(z)}}=\psi_{1}-\gamma, \\
& \phi_{2}=z^{N-1} \theta_{0} \theta_{1} \overline{a_{1}(z)}=\phi_{1}-\delta .
\end{aligned}
$$

Then $\mathbf{T}_{\mathbf{1}}=\left(T_{\phi_{1}}, T_{\psi_{1}}\right)$ is hyponormal if and only if $\mathbf{T}_{\mathbf{2}}=\left(T_{\phi_{2}}, T_{\psi_{2}}\right)$ is hyponormal. Since $\phi_{2}$ is analytic and $\psi_{2}$ is not analytic, by Corollary 4.2,

$$
\overline{z^{N-1} \theta_{0} \theta_{1}} z^{L-1} \theta_{0} \theta_{2}=z^{l-1} \Delta_{1} \overline{\Delta_{2}}
$$

for some inner functions $\Delta_{1}$ and $\Delta_{2}$, and $z^{l-1} \Delta_{1}$ and $\Delta_{2}$ are coprime. In particular, we have $L-1-(N-1) \geq l-1$.

Now $l>1$ implies that $L-1 \geq N-1+l-1 \geq N$. Note that

$$
\begin{aligned}
& \left(\begin{array}{cc}
I & 0 \\
0 & T_{z}^{*}
\end{array}\right)[\mathbf{T}, \mathbf{T}]\left(\begin{array}{cc}
I & 0 \\
0 & T_{z}
\end{array}\right) \\
& =\left(\begin{array}{cc}
H_{\overline{\phi_{+}}}^{*} H_{\overline{\phi_{+}}}-H_{\overline{\phi_{-}}}^{*} H_{\overline{\phi_{-}}} & H_{\overline{\phi_{+}}}^{*} H_{z \overline{\psi_{+}}} \\
H_{z \overline{\psi_{+}}}^{*} H_{\overline{\phi_{+}}} & H_{z \overline{\psi_{+}}}^{*} H_{z \overline{\psi_{+}}}-H_{z \overline{\psi_{-}}}^{*} H_{z \overline{\psi_{-}}}
\end{array}\right)
\end{aligned}
$$


is positive. Therefore by Lemma 2.1

$$
\begin{aligned}
& \left|\left(H_{z \overline{\psi_{+}}}^{*} H_{\overline{\phi_{+}}} x, y\right)\right|^{2} \\
& \leq\left(\left(H_{\overline{\phi_{+}}}^{*} H_{\overline{\phi_{+}}}-H_{\overline{\phi_{-}}}^{*} H_{\overline{\phi_{-}}}\right) x, x\right)\left(\left(H_{z \overline{\psi_{+}}}^{*} H_{z \overline{\psi_{+}}}-H_{z \overline{\psi_{-}}}^{*} H_{z \overline{\psi_{-}}}\right) x, x\right)
\end{aligned}
$$

for all $x, y \in H^{2}$. In particular,

$$
\left.\left|\left(H_{\overline{\phi_{+}}} x, H_{z \overline{\psi_{+}}} y\right)\right|^{2} \leq\left(\left(H_{\overline{\phi_{+}}}^{*} H_{\overline{\phi_{+}}}-H_{\overline{\phi_{-}}}^{*} H_{\overline{\phi_{-}}}\right) x, x\right)\right)\left(H_{z \overline{\psi_{+}}} y, H_{z \overline{\psi_{+}}} y\right) .
$$

It is easy to see that

$$
\operatorname{Range}\left(H_{z \overline{\psi_{+}}}\right) \supset \mathcal{H}\left(z^{L-1}\right) \supset \mathcal{H}\left(z^{N}\right)
$$

and $H_{\overline{\phi_{+}}}$maps $\theta_{0} \theta_{1} H^{2}$ onto $\mathcal{H}\left(z^{N}\right)$. Therefore

$$
\left|\left\langle H_{\overline{\phi_{+}}} x, h\right\rangle\right|^{2} \leq\left[\left(H_{\overline{\phi_{+}}} x, H_{\overline{\phi_{+}}} x\right)-\left(H_{\overline{\phi_{-}}} x, H_{\overline{\phi_{-}}} x\right)\right](h, h)
$$

for all $x \in \theta_{0} \theta_{1} H^{2}, h \in \mathcal{H}\left(z^{N}\right)$. This implies that $H_{\overline{\phi_{-}}} x=0$ for all $x \in \theta_{0} \theta_{1} H^{2}$, which is a contradiction. Therefore $l=1$. This completes the proof of the theorem for $n=1$.

Assume the theorem is true for $n-1(n \geq 2)$. Let

$$
b(z)=b_{0}+b_{1} z+\cdots+b_{n-1} z^{n-1}, \quad d(z)=d_{0}+d_{1} z+\cdots+d_{l-1} z^{l-1} .
$$

Note that

$$
\begin{aligned}
& \left(\begin{array}{cc}
T_{z}^{*} & 0 \\
0 & T_{z}^{*}
\end{array}\right)[\mathbf{T}, \mathbf{T}]\left(\begin{array}{cc}
T_{z} & 0 \\
0 & T_{z}
\end{array}\right) \\
& =\left(\begin{array}{cc}
H_{z \overline{\phi_{+}}}^{*} H_{z \overline{\phi_{+}}}-H_{z \overline{\phi_{-}}}^{*} H_{z \overline{\phi_{-}}} & H_{z \overline{\phi_{+}}}^{*} H_{z \overline{\psi_{+}}}-H_{z \overline{\psi_{-}}}^{*} H_{z \overline{\phi_{-}}} \\
H_{z \overline{\psi_{+}}}^{*} H_{z \overline{\phi_{+}}}-H_{z \overline{\phi_{-}}}^{*} H_{z \overline{\psi_{-}}} & H_{z \overline{\psi_{+}}}^{*} H_{z \overline{\psi_{+}}}-H_{z \overline{\psi_{-}}}^{*} H_{z \overline{\psi_{-}}}
\end{array}\right)=\left[\mathbf{T}_{\mathbf{1}}, \mathbf{T}_{\mathbf{1}}\right],
\end{aligned}
$$

where $\mathbf{T}_{\mathbf{1}}=\left(T_{\phi_{1}}, T_{\psi_{1}}\right)$ and

$$
\phi_{1}=P\left(\bar{z} \phi_{+}\right)+(I-P)\left(\overline{z \phi_{-}}\right), \quad \psi_{1}=P\left(\bar{z} \psi_{+}\right)+(I-P)\left(z \overline{\psi_{-}}\right) .
$$

It is easy to see that

$$
\begin{array}{ll}
\left(\phi_{1}\right)_{+}=z^{N-1} \theta_{0} \theta_{1} \overline{a_{11}(z)}, & \left(\phi_{1}\right)_{-}=z^{n-1} \overline{b_{11}(z)} \\
\left(\psi_{1}\right)_{+}=z^{L-1} \theta_{0} \theta_{2} \overline{c_{11}(z)}, & \left(\psi_{1}\right)_{-}=z^{l-1} \overline{d_{11}(z)},
\end{array}
$$

where $a_{11}(z) \in \mathcal{H}\left(z^{N-1} \theta_{0} \theta_{1}\right), c_{11}(z) \in \mathcal{H}\left(z^{N-1} \theta_{0} \theta_{2}\right)$ and

$$
b_{11}(z)=b_{0}+b_{1} z+\cdots+b_{n-2} z^{n-2}, \quad d_{11}(z)=d_{0}+d_{1} z+\cdots+d_{l-2} z^{l-2} .
$$

By the induction assumption for $n-1$, we have $N-1=L-1, n-1=l-1$, and $b_{11}(z)=\alpha d_{11}(z)$ for some constant $\alpha$. By Lemma 5.1, $\left(T_{\phi-\alpha \psi}, T_{\psi}\right)$ is hyponormal. Note that

$$
\begin{aligned}
b(z)-\alpha d(z) & =\left(b_{11}(z)+b_{n-1} z^{n-1}\right)-\alpha\left(d_{11}(z)+d_{n-1} z^{n-1}\right) \\
& =b_{n-1} z^{n-1}-\alpha d_{n-1} z^{n-1} .
\end{aligned}
$$

If $b_{n-1} \neq \alpha d_{n-1}$, then $(\phi-\alpha \psi)_{-}=z\left(\overline{b_{n-1}}-\alpha \overline{d_{n-1}}\right)$. By the previous argument, we have $\psi_{-}=z \bar{\beta}$ for some constant $\beta$. But $\psi_{-}=z^{l} \overline{d(z)}$ and $l=n \geq 2$. This is a contradiction. Therefore $b(z)=\alpha d(z)$. Now by Proposition 5.2 , we conclude that

$$
P_{\mathcal{H}\left(z^{n}\right)}\left[\theta_{2} a(z)\right]=\alpha P_{\mathcal{H}\left(z^{n}\right)}\left[\theta_{1} c(z)\right]
$$

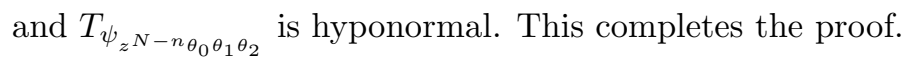


Let $\phi_{i} \in L^{\infty}$ for $i=1,2, \cdots, n$ and let $\psi \in L^{\infty}$. The next result follows easily from the previous theorem and Theorem 5.3 ,

Theorem 6.6. Assume that the $\left(\phi_{i}\right)_{-}, i=1, \cdots, n$, are all analytic polynomials. Write

$$
\begin{aligned}
\left(\phi_{i}\right)_{+} & =z^{N_{i}} \Delta_{i} \theta_{i} \overline{a_{i}(z)}, \quad\left(\phi_{i}\right)_{-}=z^{n_{i}} \overline{b_{i}(z)}, \quad i=1, \cdots, n, \\
\psi_{+} & =z^{L} \Delta_{0} \overline{c(z)}=z^{L} \Delta_{i} \delta_{i} \overline{c(z)}, \quad \psi_{-}=z^{l} \overline{d(z)},
\end{aligned}
$$

where $a_{i} \in \mathcal{H}\left(z^{N_{i}} \Delta_{i} \theta_{i}\right), b_{i} \in \mathcal{H}\left(z^{n_{i}}\right) c \in \mathcal{H}\left(z^{L} \Delta_{0}\right), d \in \mathcal{H}\left(z^{l}\right), \Delta_{i}(0) \theta_{i}(0) \delta_{i}(0) \neq 0$, and $\theta_{i}$ and $\delta_{i}$ are coprime. Then $\mathbf{T}=\left(T_{\phi_{1}}, \cdots, T_{\phi_{n}}, T_{\psi}\right)$ is hyponormal if and only if

$$
\begin{gathered}
N_{i}=L, n_{i}=l, \quad b_{i}(z)=\alpha_{i} d(z), \\
P_{\mathcal{H}\left(z^{l}\right)}\left[\delta_{i} a_{i}(z)\right]=\alpha_{i} P_{\mathcal{H}\left(z^{l}\right)}\left[\theta_{i} c(z)\right],
\end{gathered}
$$

$i=1, \cdots, n$, for some constants $\alpha_{i}$, and $T_{\psi_{z L-l_{\theta \Delta_{0}}}}$ is hyponormal, where $\theta$ is the least common multiple of $\theta_{1}, \cdots, \theta_{n}$ and

$$
\psi_{z^{L-l} \theta \Delta_{0}}=P\left(\overline{z^{L-l} \theta \Delta_{0}} \psi_{+}\right)+\overline{\psi_{-}} .
$$

Remark. The above theorem gives a complete characterization for the hyponormality of $\mathbf{T}=\left(T_{\phi_{1}}, \cdots, T_{\phi_{n}}, T_{\psi}\right)$ when only one of the symbols is a trigonometric polynomial. We note that if $\psi$ is a trigonometric polynomial, say

$$
\psi_{+}=z^{L} \overline{c(z)}, \quad \psi_{-}=z^{l} \overline{d(z)},
$$

where $c(z) \in \mathcal{H}\left(z^{L}\right), d(z) \in \mathcal{H}\left(z^{l}\right)$, the hyponormality of $\left(T_{\phi}, T_{\psi}\right)$ will force $\phi$ to take a certain form. Namely, by Lemma 6.2

$$
\operatorname{Ker}\left(H_{\overline{\psi_{-}}}\right)=z^{l} H^{2} \supset \operatorname{Ker}\left(H_{\overline{\phi_{+}}}\right), \quad \operatorname{Ker}\left(H_{\overline{\phi_{-}}}\right) \supset z^{L} H^{2}=\operatorname{Ker}\left(H_{\overline{\psi_{+}}}\right) .
$$

Therefore

$$
\phi_{+}=z^{N} \theta \overline{a(z)}, \quad \phi_{-}=z^{n} \overline{b(z)},
$$

where $N \geq l, n \leq L, c \in \mathcal{H}\left(z^{N} \theta\right)$ and $d \in \mathcal{H}\left(z^{n}\right)$.

We conclude the paper with the following corollary.

Corollary 6.7 (Curto and Lee, 2001). Let $\mathbf{T}=\left(T_{\phi_{1}}, \cdots, T_{\phi_{n}}\right)$ be an n-tuple of trigonometric Toeplitz operators. Then the following three statements are equivalent.

i) The tuple $T$ is hyponormal.

ii) Every subpair of $T$ is hyponormal.

iii) The symbols $\phi_{i}$ possess the following properties:

a) All non-analytic trigonometric polynomials $\phi_{i}$ are of the form

$$
\phi_{i}(z)=\sum_{k=-m}^{N} a_{k} z^{k},
$$

where $a_{-m}$ and $a_{N}$ are nonzero, every $T_{\phi_{i}}$ is hyponormal, and for every pair $\left\{\phi_{i}, \phi_{j}\right\}$ $(i \neq j)$ we have $\phi_{i}-c \phi_{j}=\sum_{k=0}^{N-m} d_{k} z^{k}$ for some constants $c$ and $d_{0}, \cdots, d_{m}$.

b) $\max \left\{\right.$ Degree $\left(\phi_{i}\right): \phi_{i}$ is an analytic polynomial $\} \leq N-m$. 
Proof. i) implies ii) is evident. We next show ii) implies iii). Without loss of generality, assume

$$
\phi_{n}(z)=\sum_{k=-m}^{N} a_{k} z^{k}=\left(\phi_{n}\right)_{+}+\overline{\left(\phi_{n}\right)_{-}},
$$

where $a_{-m}$ and $a_{N}$ are nonzero. Since the commutator of $T_{\phi_{n}}$ does not depend on the constant term $a_{0}$, we assume $a_{0}=0$. Set

$$
a(z)=\overline{a_{N}}+\overline{a_{N-1}} z+\cdots+\overline{a_{1}} z^{N-1}, \quad b(z)=\overline{a_{-m}}+\overline{a_{-m+1}} z+\cdots+\overline{a_{-1}} z^{m-1} .
$$

It is easy to see that

$$
\left(\phi_{n}\right)_{+}=z^{N} \overline{a(z)}, \quad\left(\phi_{n}\right)_{-}=z^{m} \overline{b(z)} .
$$

Let $1 \leq i \leq n-1$. Assume $\left(T_{\phi_{i}}, T_{\phi_{n}}\right)$ is hyponormal. If $\phi_{i}$ is an analytic polynomial of degree $l$, then $\operatorname{Ker}\left(H_{\overline{\phi_{i}}}\right)=z^{l} H^{2}$. Since the greatest common divisor of $z^{l}$ and $z^{N}$ is $z^{k}$, where $k$ is the minimum of $l$ and $N$, by Corollary 4.2, $z^{k}$ is a factor of $z^{N-m}$. Therefore $l \leq N-m$. If $\phi_{i}$ is a non-analytic trigonometric polynomial, write

$$
\left(\phi_{i}\right)_{+}=z^{L} \overline{c(z)}, \quad\left(\phi_{i}\right)_{-}=z^{l \overline{d(z)},}
$$

where $c \in \mathcal{H}\left(z^{L}\right)$ and $d \in \mathcal{H}\left(z^{l}\right)$. By Theorem 6.5, we have $N=L, m=l$, and

$$
b(z)=\alpha d(z), \quad P_{\mathcal{H}\left(z^{m}\right)}[a(z)]=\alpha P_{\mathcal{H}\left(z^{m}\right)}[c(z)] .
$$

This is exactly the same as

$$
\phi_{n}-\alpha \phi_{i}=\sum_{k=0}^{N-m} d_{k} z^{k}
$$

for some constants $\alpha$ and $d_{0}, \cdots, d_{N-m}$. The implication of iii) to i) follows from Corollary 5.4. This completes the proof.

\section{REFERENCES}

[1] M. B. Abrahamse, Subnormal Toeplitz operators and functions of bounded type, Duke Math. J. 43(1976), 597-604. MR 55:1126

[2] I. Amemiya, T. Ito and T. K. Wong, On quasinormal Toeplitz operators, Proc. Amer. Math. Soc. 50(1975), 254-258. MR 53:14200

[3] A. Athavale, On joint hyponormality of operators, Proc. Amer. Math. Soc. 103(1988), 417423. MR 89f: 47033

[4] S. Axler, S.-Y. A. Chang, D. Sarason, Product of Toeplitz operators, Integral Equations and Operator Theory 1 (1978), 285-309. MR 80d:47039

[5] J. Bram, Subnormal operators, Duke Math. J. 22(1955), 75-94. MR 16:835a

[6] A. Brown and P. R. Halmos, Algebraic properties of Toeplitz operators, J. Reine Angew. Math. 213(1963), 89-102. MR 28:3350

[7] J. B. Conway and W. Szymanski, Linear combination of hyponormal operators, Rocky Mountain J. Math. 18(1988), 695-705. MR 90a:47059

[8] C. C. Cowen, Hyponormality of Toeplitz operators, Proc. Amer. Math. Soc. 103(1988), 809-812. MR 89f:47038

[9] C. C. Cowen, More subnormal Toeplitz operators, J. Reine Angew. Math. 351(1986), 215219. MR 87h:47063

[10] C. C. Cowen and J. J. Long, Some subnormal Toeplitz operators, J. Reine Angew. Math. 351(1984), 216-220. MR 86h:47034.

[11] R. E. Curto, Quadratically hyponormal weighted shifts, Integral Equation and Operator Theory 13(1990), 49-66. MR 90k:47061 
[12] R. E. Curto, Joint hyponormality: A bridge between hyponormality and subnormality, Proc. Sympos. Pure Math., vol 51 part II (W. B. Arveson and R. G. Douglas eds.), 1990, 69-91. MR 91k:47049

[13] R. E. Curto and W. Y. Lee, Joint hyponormality of Toeplitz pairs, Memoirs of Amer. Math. Soc., Vol. 150, No. 712, March 2001. CMP 1810770

[14] R. E. Curto, P. S. Muhly and J. Xia, Hyponormal pairs of commuting operators, Operator Theory: Advances and Application (I. Gohberg, J. W. Helton and L. Rodman eds.), Vol 35(1988), 1-22. MR 90m:47037

[15] P. Fan, A note on hyponormal weighted shift, Proc. Amer. Math. Soc. 92(1984), 271-272. MR 86c:47037

[16] D. R. Farenick and W. Y. Lee, Hyponormality and spectra of Toeplitz operators, Trans. Amer. Math. Soc. 348(1996), 4153-4174. MR 97k:47027

[17] D. R. Farenick and R. McEachin, Toeplitz operators hyponormal with the unilateral shift, Integral Equation and Operator Theory 22(1995), 273-280. MR 96f:47042

[18] C. Foias and A. Frazho, The commutant lifting approach to interpolation problem, Operator Theory: Adv. Appl., vol 44, Birkḧauser-Verlag, Boston, 1990. MR 92k:47033

[19] C. Gu, A generalization of Cowen's characterization of hyponormal Toeplitz operators, J. Funct. Anal. 124(1994), 135-148. MR 95j:47034

[20] P. R. Halmos, Ten problems in Hilbert space, Bull. Amer. Math. Soc. 76(1970), 887-933. MR 42:5066

[21] S. McCullough and V. I. Paulsen, A note on joint hyponormality, Proc. Amer. Math. Soc. 107(1989), 187-195. MR 90a:47062

[22] T. Nakazi and K. Takahashi, Hyponormal Toeplitz operators and extremal problems of Hardy spaces, Trans. Amer. Math. Soc. 338(1993), 753-767. MR 93j:47040

[23] I. Schur, On power series which are bounded in the interior of the unit circle I, J. Reine Angew. Math. 147(1917), 205-232.

[24] Sun Shunhua, On Toeplitz operators in the $\Theta$-class, Scientia Sinica (Series A) 28(1985), 235-241. MR 86g:47029

Department of Mathematics, California Polytechnic State University, San Luis Obispo, CALifornia 93407

E-mail address: cgu@calpoly.edu 